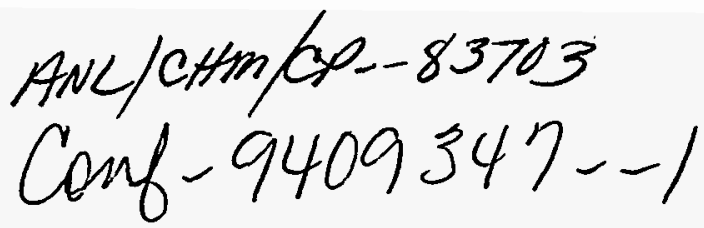

Metal-Ligand Interactions: Structure and Reactivity, N. Russo, Ed., Kluwer Academic Pub., Dordrecht, The Netherlands

\title{
Theoretical Dynamical Studies of Metal Clusters and Cluster-Ligand Systems*.
}

\author{
J. Jellinek \\ Chemistry Division \\ Argonne National Laboratory \\ Argonne, IL 60439 USA
}

\section{DISCLAIMER}

This report was prepared as an account of work sponsored by an agency of the United States Government. Neither the United States Government nor any agency thereof, nor any of their employees, makes any warranty, express or implied, or assumes any legal liability or responsibility for the accuracy, completeness, or usefulness of any information, apparatus, product, or process disclosed, or represents that its use would not infringe privately owned rights. Reference herein to any specific commercial product, process, or service by trade name, trademark, manufacturer, or otherwise does not necessarily constitute or imply its endorsement, recommendation, or favoring by the United States Government or any agency thereof. The views and opinions of authors expressed herein do not necessarily state or reflect those of the United States Government or any agency thereof.

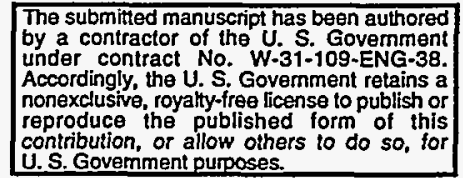

*Work performed under the auspices of the Office of Basic Energy Sciences, Division of Chemical Science, US-DOE under contract number W-31-109-ENG-38. 


\section{DISCLAIMER}

Portions of this document may be illegible in electronic image products. Images are produced from the best available original document. 


\title{
THEORETICAL DYNAMICAL STUDIES OF METAL CLUSTERS AND CLUSTER-LIGAND SYSTEMS
}

\author{
J. JELLINEK \\ Chemistry Division \\ Argonne National Laboratory \\ Argonne, IL 60439, USA
}

\section{Introduction}

Metal-ligand interactions constitute the subject of a variety of disciplines that include organo- and inorganometallic chemistry, surface science, and the rapidly developing field of cluster research. In what follows we use the term cluster to designate a cohesive group of like atoms (molecules), i.e., bare ("neet", "naked") clusters. More generally, the term is also used for organo- and inorganometallic compounds, i.e., ligated clusters. Although the approaches and techniques used by the various disciplines to study metal-ligand interactions are quite different, many of the central subjects and issues are common for them. The common subjects include possible geometric structures and isomeric forms, structural (isomerization) transitions, stability, fluxionality, structure-reactivity correlation (or lack of it), role of coordination, etc. However, the precise interpretation of these issues and the details emphasized by the different disciplines are dictated by the nature of the objects studied and may not, therefore, be identical. For example, questions regarding structures, isomerization transitions, fluxionality or even melting of metal clusters refer to the state and properties of the metal network itself. The same questions, when asked in connection with organo- and inorganometallic compounds, often refer to the arrangements and rearrangements of the ligands attached to a metal framework of a fixed structure. Of course, when required, the state of and changes in the metal framework are considered as well. The fields of metalcontaining molecular compounds, surface science, and physics and chemistry of clusters furnish complementary information on a broad variety of metal-ligand systems. A comprehensive understanding of the nature and properties of these systems, as defined by the type and number of metal atoms and ligands involved, can be achieved only through a mutual awareness of and continuing progress in all of these research areas.

The field of clusters grew out of an effort aimed at understanding and describing surface and bulk properties of materials on a microscopic (atomic) level. Therefore the first theoretical studies of metal clusters assumed atomic arrangements consistent with different crystal lattices. It was soon realized, 
however, that the properties of clusters, especially those of small and intermediate sizes, are, in general, different from the properties of bulk matter. Thus, for example, the most stable geometric forms of clusters in these size ranges are usually not slabs of crystal lattices. Finite size effects exhibit themselves also in the electronic structure. Change of cluster properties with their size and the way these properties evolve into those of bulk materials is a complex issue. The minimal size of a cluster at which a chosen physical property attains its bulk value depends on the property of interest. Moreover, the size-evolution of the properties is not necessarily monotonic or even unique. All this makes the relationship between clusters and bulk materials not as straightforward as originally thought. At present, it appears to be uniformly accepted that atomic (molecular) clusters form a distinct phase of matter that is intermediate between the traditional gas and condensed phases. The interest in diverse and often unexpected and intricate properties of this phase and the everincreasing recognition of its importance in natural phenomena and in technological applications established cluster research as a field in its own right, with its own subjects, techniques, and methodologies. It is hardly an exaggeration to characterize the recent surge and progress in theoretical and experimental research in this field as phenomenal [1-7]. The enthusiasm of its practitioners is fueled by the realization that what has been "seen and touched" is still only the "tip of the iceberg".

Theoretical studies of clusters involve analytical modelling and numerical approaches. The latter include electronic structure calculations and molecular dynamics (MD) and Monte Carlo (MC) simulations. Electronic structure calculations utilize different levels of ab initio quantum chemistry techniques, density functional theory, and more approximate approaches, such as the tightbinding model. These calculations are carried out for fixed nuclear geometries. For bare clusters they provide information on energies, relative stabilities of different isomeric forms, and optical and magnetic properties. For cluster-ligand systems they furnish bonding attributes such as binding energies and preferred binding arrangements and sites. When repeated on a grid of nuclear configurations, they characterize parts of a potential energy surface describing interatomic interactions. The degree of adequacy and reliability of the results depends on the level of the electronic structure treatment. Accurate values of energy pose a computationally very demanding task even for clusters of modest sizes for most materials, and even on the most advanced computers. Therefore, only very limited parts (e.g., a cut) of potential energy surfaces can be mapped out with sufficient accuracy through electronic structure calculations.

Most MD and MC simulations use semiempirical potentials for mimicking the interatomic interactions. The computational efficiency of these potentials allows for characterization of the state of clusters and cluster-molecule systems through adequate sampling of their atomic phase or configuration spaces. Adequate sampling requires performing long and/or many $\mathrm{MD}$ simulation runs or a large number of MC steps. From these one derives time- and ensembleaveraged structural and dynamical properties of the systems studied. An added advantage of $\mathrm{MD}$ studies is that they also allow one to follow the time-evolution 
of systems and thereby to understand and to describe the mechanisms of processes taking place. An important issue is the quality, or degree of reliability, of the potential energy surfaces used. For systems, such as, e.g., clusters of noble gas atoms, in which the (van der Waals) bonding can be represented sufficiently accurately as a sum of all the pairwise interactions, the quality of the potential is defined by the quality of description of the interaction between two atoms. The situation is different for metal clusters, in which delocalization of electrons leads to an important role of the so-called manybody effects. Adequate modelling of these effects remains a challenging task.

A decade ago Car and Parinello (CP) [8] formulated a MD scheme in which density functional calculations replace the semiempirical potentials. The time consuming density functional calculations are repeated only "as needed", i.e., as new nuclear configurations are generated by the coupled (quantal) electronic and (classical) nuclear dynamics. The CP scheme and its various modifications and generalizations [9-11], commonly labeled as first principles (or ab initio) MD's, have been applied with success to a broad variety of systems including clusters, surfaces, and bulk matter. Most applications are directed at structural and energetic characteristics. For example, in studies of clusters, ab initio MD's are very efficient in finding different isomeric forms, including the energetically most stable ones, even when these forms are of low symmetry and therefore would be difficult, if not impossible, to guess a priori. However, use of these MD's in studies of dynamical properties of systems remains very limited $[10,11]$. The reason is that, as mentioned above, a meaningful dynamical analysis can be carried out only if the ensemble of the sampled microscopic states is sufficiently representative. The number of configurations in such an ensemble, i.e., the number of times a first principles electronic structure calculation has to be performed, makes the computational task for most systems prohibitively costly notwithstanding the phenomenal recent advances in computational methodologies (e.g., parallel processing) and in computer hardware. To make the computational task more feasible, the electronic structure parts of first principles MD's incorporate approximations, such as the local density approximation in the density functional theory, core pseudopotentials, neglect or only approximate inclusion of electron correlation effects, truncation of basis sets, etc. Whereas some physical characteristics such as, e.g., equilibrium geometries (atomic packings) and stability ordering of isomers, are usually (but not necessarily) less sensitive to these approximations, others, such as absolute magnitudes of cohesive energy and equilibrium interatomic distances, often show a high degree of sensitivity. One should be aware of the fact that these approximations may introduce errors and/or uncertainties that are comparable or even exceed those associated with semiempirical potentials, thereby defeating the very purpose of the ab initio MD's which is to provide more accurate results. In each specific case an analysis of implications and consequences of the approximations involved is warranted. A highly nontrivial but very important question, which has hardly been addressed, can be formulated as follows: What is the necessary and sufficient degree of accuracy of a description of the interatomic interactions that 
is needed for extraction of a specified set of physical properties of a system at a desired level of detail and confidence? With further advances in computational speedup the role of first principles MD studies will increase. Most theoretical dynamical and MC treatments, however, will have to continue to rely on ever more accurate semiempirical potentials. Rather then contrasting the ab initioand potential-based MD approaches, one should view them as complementary. In fact, data obtained within even limited ab initio MD studies, which utilize sufficiently accurate electronic structure calculations, can be used for testing and improving the quality of semiempirical potentials.

In the following sections we present samples and a discussion of types and results of dynamical studies of metal clusters and cluster-ligand systems. In Section 2 we recap the essence of trajectory simulation techniques. In Section 3 a brief description of the potential energy functions used to describe the intracluster and cluster-ligand interactions is given together with some pertinent details of the computational procedure. Results on structures of metal clusters of different sizes are presented in Section 4. Section 5 contains a discussion of the evolution of intracluster dynamics with the cluster energy. The $\mathrm{D}_{2}+\mathrm{Ni}_{13}$ collision system is analyzed in Section 6 as a paradigm of cluster-molecule interactions. A brief summary is given in Section 7.

\section{Molecular Dynamics Techniques}

Classically, the mechanical state of a system consisting of $\mathbf{n}$ discrete particles (bodies) considered as point masses $m_{i}(i=1, \ldots, n)$ is completely defined by a set of generalized coordinates and velocities (Lagrangian formulation) or, alternatively, generalized coordinates and conjugate momenta (Hamiltonian formulation) of all the particles [12]. Knowledge of these quantities allows for determination of all other mechanical characteristics of systems. To be specific, we shall use the Cartesian coordinates $r_{i}$, velocities $v_{i}$, and momenta $\mathrm{p}_{i}$. Determinism of classical mechanics means that the state of a system at any future time $t>t_{0}$ is uniquely defined by its state at (an arbitrary) initial time $t_{0}$ and the forces acting on its constituent particles. For closed (isolated) systems the forces are defined solely by the interparticle interactions. These interactions are represented by the potential energy function $V$, which we assume, as is indeed the case in most situations, to depend only on the particle coordinates $r_{i}$. The time evolution of a state is defined by Lagrange's equations

$$
\frac{\mathrm{d}}{\mathrm{dt}} \frac{\partial \mathscr{L}}{\partial \mathrm{v}_{\mathrm{i}}}=\frac{\partial \mathscr{L}}{\partial \mathrm{r}_{\mathrm{i}}}
$$

where the Lagrangian $\mathcal{L}$ is defined as

$$
\mathcal{L}=\sum_{\mathrm{i}=1}^{\mathrm{n}} \frac{1}{2} \mathrm{~m}_{\mathrm{i}} \mathrm{v}_{\mathrm{i}}^{2}-\mathrm{V}\left(\mathrm{r}_{1}, \ldots, \mathrm{r}_{\mathrm{n}}\right)
$$


or, alternatively, by Hamilton's equations

$$
\begin{gathered}
\frac{d r_{i}}{d t}=\frac{\partial \mathscr{H}}{\partial p_{i}}, \\
\frac{d p_{i}}{d t}=-\frac{\partial \mathscr{H}}{\partial r_{i}},
\end{gathered}
$$

where the Hamiltonian $\&$ is given by

$$
\mathscr{H}=\sum_{\mathrm{i}=1}^{n} \frac{p_{i}^{2}}{2 m_{i}}+V\left(r_{1}, \ldots, r_{n}\right) .
$$

(Boldface is used to designate vectors). Substitution of Eq. (2) into Eqs. (1) leads to Newton's form of equations of motion

$$
m_{i} \frac{d v_{i}}{d t}=-\frac{\partial V}{\partial r_{i}}
$$

or

$$
\mathrm{m}_{\mathrm{i}} \frac{\mathrm{d}^{2} \mathbf{r}_{\mathrm{i}}}{\mathrm{dt}^{2}}=-\frac{\partial \mathrm{V}}{\partial \mathbf{r}_{\mathrm{i}}}
$$

The right-hand side of Eqs. (5') is the force acting on particle i. For more general discussions and analyses of the connection between the Lagrangian and Hamiltonian formulations the reader is referred to one of the numerous books on classical mechanics.

The essence of the MD technique is in integrating numerically the set of $n$ ( $3 n$ in scalar form) second order differential equations (5') or $2 \mathrm{n}$ (6n in scalar form) first order differential equations (3). The task is accomplished by rewriting these equations in terms of finite differences and solving them on a discrete time grid by means of computers [13]. Different numerical integration routines can be used for this purpose, and the choice of a specific one, as well as of the integration grid, are governed by considerations such as accuracy and efficiency. With continuous advances in computational speed, systems of everlarger sizes become amenable to MD simulations.

We consider in what follows, unless otherwise specified, Hamiltonian dynamics of systems of discrete point masses and the corresponding $\mathrm{MD}$ techniques. Equations (3) define continuous time-reversible trajectories in a $6 n$-dimensional phase space. However, the manifold actually sampled by trajectories may be, and usually is, of lower dimensionality because of the constraints introduced by the applicable integrals of motion (conserved quantities). Total energy and total linear and angular momenta are examples of well-known and often applicable (but not the only possible) integrals of motion. 
Most numbers can be represented on computers only in a truncated, roundedoff form. The accumulation of the resulting round-off errors and the approximations involved in the finite difference representation of Eqs. (3) have a number of consequences. One of these is that computer-generated trajectories retain the property of time-reversibility only for certain, usually not too long, propagation times. This is especially true for the so-called chaotic dynamics, which are highly sensitive to initial conditions. For studies of many properties, however, the lack of complete time-reversibility is of only minor or no significance at all. Another consequence is the drift in the values of quantities that, as defined by the exact dynamics, are integrals of motion. The effect of this drift on the validity of the simulations may not be negligible. The drifts can be kept within predefined tolerance limits through appropriate adjustment(s) of the integration step. An optimal compromise between accuracy and efficiency of an $M D$ simulation can be achieved through use of a variable time step and/or an integrator of an appropriate order. Detailed discussion of these and other related issues can be found in [13].

In the remainder of this section we turn briefly to more recent developments. The first one deals with the selection of initial conditions that are compatible with specified values of total energy $E$ and total angular momentum $L$ of an arbitrary n-body system, as defined in its center-of-mass (COM) laboratoryoriented system of coordinates. In most $\mathrm{MD}$ simulations it is assumed that $\mathrm{L}=0$, which considerably simplifies the choice of the initial conditions. It may be surprising that a general solution to this problem has not been given until recently [14-16]. The nontrivial nature of the task becomes transparent through the realization that it is intimately connected to the problem of definition of rotational dynamics in nonrigid systems. The general solution to the problem of initial conditions emerges as a byproduct of a new definition of rotational dynamics $[14,15]$, which is different from the ones implied by the traditionally used principal-axes and Eckart body-fixed (rotating) frames [17].

One should be aware of the fact that not all pairs of $E$ and $L$ are compatible. The total energy $E$ of a system with a specified total angular momentum $L$ and a defined interaction potential $\mathrm{V}$ has a well-defined lower limit [14,15]. Assuming that an acceptable pair of $E$ and $L$ has been chosen, the task is to define a set of initial COM coordinates $r_{i}^{o}$, and momenta $p_{i}^{o}, i=1, \ldots, n$, such that

$$
\begin{gathered}
\sum_{i=1}^{n} \frac{p_{i}^{o^{2}}}{2 m_{i}}+V\left(r_{1}^{o} \ldots, r_{n}^{o}\right)=E, \\
\sum_{i=1}^{n} r_{i}^{o} \times p_{i}^{o}=L,
\end{gathered}
$$




$$
\begin{aligned}
& \sum_{i=1}^{n} r_{i}^{0}=0, \\
& \sum_{i=1}^{n} p_{i}^{o}=0 .
\end{aligned}
$$

First, one selects an arbitrary set of coordinates $r_{i}^{o}, i=1, \ldots, n-1$, and calculates $r_{\mathrm{n}}^{0}$ from Eq. (8). The chosen configuration defines the potential energy $V\left(r_{1}^{o}, \ldots, r_{n}^{o}\right)$ of the system. Then one computes the angular velocity $\omega$ of the system in accordance with $[14,15]$

$$
\omega\left(r_{1}^{0}, \ldots, r_{n}^{o} ; L\right)=\overleftrightarrow{I}^{-1}\left(r_{1}^{o}, \ldots, r_{n}^{o}\right) \cdot L,
$$

where $\overline{\mathrm{I}}^{-1}$ is the inverse of the tensor of inertia of the chosen configuration. In terms of its components the tensor of inertia $\overrightarrow{\mathrm{I}}\left(\mathrm{r}_{1}, \ldots, \mathrm{r}_{n}\right)$ is a $3 \times 3$ matrix with elements

$$
I_{\alpha \alpha}=\sum_{i=1}^{n} m_{i}\left(r_{i}^{2}-\alpha_{i}^{2}\right), \quad I_{\alpha \beta}=-\sum_{i=1}^{n} m_{i} \alpha_{i} \beta_{i}, \quad \alpha, \beta(\neq \alpha)=x, y, z,
$$

where $x_{i}, y_{i}$, and $z_{i}$ are the components of $r_{i}$. It is clear from Eq. (10) that the angular velocity is defined to "carry" the entire total angular momentum $L$. Next, one calculates the rotational energy $\mathrm{E}_{\mathrm{r}}\left(\mathrm{r}_{1}^{\mathrm{o}}, \ldots, \mathrm{r}_{\mathrm{n}}^{\mathrm{o}} ; \mathrm{L}\right)$ as $[14,15]$

$$
E_{r}\left(r_{1}^{o}, \ldots, r_{n}^{o} ; L\right)=\frac{1}{2} \omega\left(r_{1}^{o}, \ldots, r_{n}^{o} ; L\right) \cdot L \text {. }
$$

In accordance with the theorem proven in [14], the minimal energy of a system having the chosen configuration and angular momentum $\mathrm{L}$ is

$$
E_{\min }\left(r_{1}^{o}, \ldots, r_{n}^{o} ; L\right)=E_{r}\left(r_{1}^{o}, \ldots, r_{n}^{o} ; L\right)+V\left(r_{1}^{o}, \ldots, r_{n}^{o}\right) .
$$

Unless $E_{\min } \leq E$, the chosen configuration of the system is unacceptable and another selection must be made as described above. If $\mathrm{E}_{\min }=\mathrm{E}$, an event that hardly will happen in practice, the specification of the initial conditions is completed by assigning to the momenta the values

$$
p_{i r}^{o}=m_{i}\left(\omega \times r_{i}^{o}\right) .
$$

The subscript $r$ in $p_{i r}^{0 \prime}$ indicates that the initial momenta of the particles are entirely rotational momenta. By construction they satisfy Eq. (7). Because the

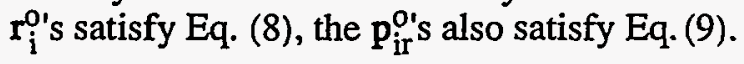


Except by accident, the choice of acceptable initial values of the coordinates leads to $E_{\min }<E$. The task is to modify the $p_{i r}^{o '} s$ defined by Eq. (14) into $p_{i}^{\text {O's }} s$ in such a way that the total angular momentum $L$ and the zero total linear momentum remain unaltered, but the total energy acquires the desired value $E$. This is achieved in the following way. Assign to the particles test initial momenta $p_{i}^{t}$ that sum up to zero but otherwise are arbitrary. This can be done in a manner specified above for the initial values of the coordinates. The total angular momentum $\mathrm{L}^{\mathrm{t}}$ defined by these momenta,

$$
L^{t}=\sum_{i=1}^{n} r_{i}^{o} \times p_{i}^{t}
$$

is, in general, different from the desired angular momentum $L$. One calculates the angular velocity $\omega^{t}$ that carries the angular momentum $L^{t}$ in accordance with

$$
\omega^{\mathrm{t}}\left(\mathrm{r}_{1}^{\mathrm{o}}, \ldots, \mathrm{r}_{n}^{\mathrm{o}} ; \mathrm{L}^{\mathrm{t}}\right)=\overrightarrow{\mathrm{I}}^{-1}\left(\mathrm{r}_{1}^{\mathrm{o}}, \ldots, \mathrm{r}_{\mathrm{n}}^{\mathrm{o}}\right) \cdot \mathrm{L}^{\mathrm{t}}
$$

and defines residual momenta $\Delta \mathbf{p}_{\mathbf{i}}^{\mathbf{t}}$,

$$
\Delta p_{i}^{t}=p_{i}^{t}-p_{i r}^{t}=p_{i}^{t}-m_{i}\left(\omega^{t} \times r_{i}^{o}\right) .
$$

The $\Delta \mathrm{p}_{\mathrm{i}}^{\mathrm{t}} \mathrm{s}$ satisfy the conditions [14]

$$
\sum_{i=1}^{n} r_{i}^{o} \times \Delta p_{i}^{t}=0
$$

and

$$
\sum_{i=1}^{n} \Delta p_{i}^{t}=0
$$

and so, obviously, do their scaled counterparts $\Delta \mathrm{p}_{\mathrm{i}}^{\mathrm{o}}$,

$$
\Delta \mathrm{p}_{\mathrm{i}}^{\mathrm{o}}=\zeta \Delta \mathrm{p}_{\mathrm{i}}^{\mathrm{t}},
$$

where the scaling factor $\zeta$ is defined as

$$
\zeta=\left\{\left[E-E_{\min }\left(r_{1}^{o}, \ldots, r_{n}^{o} ; L\right)\right] /\left[\sum_{i=1}^{n}\left(\Delta p_{i}^{t}\right)^{2} / 2 m_{i}\right]\right\}^{1 / 2}
$$


Then the momenta

$$
\mathbf{p}_{\mathrm{i}}^{0}=\Delta \mathrm{p}_{\mathrm{i}}^{0}+\mathrm{p}_{\mathrm{ir}}^{\mathrm{o}},
$$

where the $\Delta p_{i}^{o ' s}$ and $p_{i r}^{o ' s}$ are defined by Eqs. (20) and (14), respectively, satisfy Eqs. (7) and (9). Because of the choice of the scaling factor $\zeta$, Eq. (21), and the fact that

$$
\sum_{i=1}^{n} \frac{\Delta p_{i}^{o} \cdot p_{i r}^{o}}{m_{i}}=0
$$

(see [13] for proof), the $p_{i}^{0 ' s}$ defined by Eq. (22) also satisfy Eq. (6), and, therefore, together with the $\mathrm{r}_{\mathrm{i}}$ 's they form a set of the desired initial conditions. The prescription formulated above can be generalized in a straightforward way to the case when one needs to select initial coordinates and momenta compatible with predefined (internally consistent) values of $\mathrm{E}, \mathrm{L}$ and total linear momentum $\mathrm{P}$, all specified in a laboratory-fixed system of coordinates. Apart from leading to a general recipe for the initial conditions, the definition of rotational dynamics introduced in [14] allows for separate control of the total angular momentum and the internal energy of n-body systems. In particular, it leads to an efficient L-preserving quenching technique that allows one to find equilibrium structures of rotating floppy systems and to study the evolution of these structures with L [15]. It also leads to an L-dependent normal mode scheme, in which the effect of the centrifugal distortions on the fundamental frequencies of a system is taken into account exactly [18].

An important issue is the relationship between dynamics and statistical mechanics, or more precisely, between time-averaged properties of a single system and ensemble-averaged properties of a collection of such systems, all of which are in the same macrostate (e.g., have the same total energy or temperature) but are distributed over the different possible microstates (for a more detailed discussion see [19-21]). The essence of the ergodicity hypothesis is that for any physical property the two types of averaging procedures give equal values. An ensemble is specified by a probability density defined over the phase space, i.e., over the microstates. Apart from the constraints introduced by conservation laws other than that for the total energy, the dynamics discussed above simulate conditions corresponding to the microcanonical ensemble. The ergodicity assumption implies in this case that the trajectory generated by a dynamics visits the vicinity of all the accessible phase points of an energy shell with equal probability. The von Neuman-Birkhoff theorem [19,22], which establishes the conceptual foundations of the ergodic property, is, in fact, proven only for conservative systems. One still can consider the problem of generating dynamics (i.e., continuous deterministic and, at least in principle, time-reversible trajectories) that visit phase space points in accordance with probability densities other than that of the microcanonical ensemble [20]. 
A quite remarkable development is the formulation by Nose of an isothermal MD scheme [23]. Although we shall not present here any details (some of which are quite intricate $[20,21]$ ) or results, we feel that no discussion of MD techniques can be complete without mentioning it. The Nosé dynamics simulates conditions corresponding to a canonical ensemble, i.e., it defines a time evolution of a physical system with a specified temperature. Because the system samples states with different total energy, its dynamics cannot be Hamiltonian. By construction the Nosé dynamics weights every microstate (phase point) it reaches by the Boltzmann factor corresponding to that state. The issue of ergodicity then becomes the issue of ability of the Nose dynamics to reach all the relevant points of the phase space. In more practical terms the issue is that of an adequate sampling of the different energy shells on the time scale of realistic computations. It turns out [24] that the original Nosé scheme, which for some time was believed to be the only continuous deterministic dynamics capable of weighting the different phase points by the Boltzmann factor, may, in fact, not be sufficiently efficient for such a sampling. Subsequent developments include the realization that there exist infinitely many different Nosé-type dynamics [20] and the formulation of a variety of its generalizations (for a review, see [25]). The subject remains under active scrutiny, and current efforts continue to be directed at constructing Nosé-type MD's with higher sampling efficiency.

\section{Interaction Potentials and Computational Details}

The results presented and discussed in the following sections are obtained from semiempirical potentials. Here we give a brief description of these potentials together with some relevant details of the computational procedure. We consider two types of description of the interatomic interactions in metallic systems. The two are similar in that they are comprised of pairwise additive interactions and an additional term introduced to account for the many-body effects. These effects are consequences of the delocalized nature of metallic bonding. The first potential is of the so-called embedded-atom type [26]. It can be written as

$$
V_{E A}=\frac{1}{2} \sum_{i, j=1}^{n} \phi\left(r_{i j}\right)+\sum_{i=1}^{n} F\left(\bar{\rho}_{i}\right),
$$

where $\phi$ represents a pairwise (Morse) interaction, $F$ is the many-body term called embedding energy, $r_{i j}$ is the distance between atoms $i$ and $j$, and $\bar{\rho}_{i}$ is the electron density experienced by atom $i$. This density is the superposition of the electron densities $\rho_{j}\left(r_{i j}\right)$ supplied by the surrounding atoms $j(\neq i)$ at the position of atom i: 


$$
\bar{\rho}_{i}=\sum_{j(\neq i)} \rho_{j}\left(r_{i j}\right)
$$

We use Voter and Chen's parameterization of $\phi F$, and $\rho_{j}$ [27]. The values of the parameters are defined by a simultaneous fit to the measured equilibrium facecentered cubic ( $\mathrm{fcc}$ ) lattice constant, cohesive energy, bulk modulus, three cubic elastic constants, and vacancy formation energy of the fcc bulk metal of interest, as well as of the equilibrium bond length and bond energy of its diatomic molecule. For details and values of the parameters for nickel, a metal to be considered below, see [27]. Since properties of two extreme size ranges are used in the fit, the potential may be expected to be of higher degree of relevance for clusters.

The second potential is usually referred to in the literature as that of Gupta or Gupta-like type [28], although it was considered originally by Ducastelle [29]. The functional form of the attractive part of this potential is based on the second moment approximation to the tight-binding model. It is this part that incorporates all the many-body effects. The pairwise additive repulsive part is a sum of Born-Mayer terms. In reduced units of energy $\mathrm{V}^{*}$ and interatomic distances $r_{i j}^{*}$ the Gupta-like potential is written as

$$
V^{*}=\frac{1}{2} \sum_{j=1}^{n}\left\{A \sum_{i(\neq j)=1}^{n} \exp \left[-p\left(r_{i j}^{*}-1\right)\right]-\left(\sum_{i(\neq j)=1}^{n} \exp \left[-2 q\left(r_{i j}^{*}-1\right)\right]\right)^{1 / 2}\right\} \text {, }
$$

where the parameters A, p, and q depend on the metal. Below we present results for nickel and gold clusters modeled by the Gupta-like potential. For nickel we adopt the values $p=9$ and $q=3$, which have been used for transition metals [29]. For gold, fitting of measured bulk properties gives $p=10.15$ and $q=4.13$ [30]. The reduced energy $V^{*}$ and distance $r_{i j}^{*}$ are defined as

$$
\mathrm{V}^{*}=\frac{\mathrm{V}}{\mathrm{U}}, \quad \mathrm{r}_{\mathrm{ij}}^{*}=\frac{\mathrm{r}_{\mathrm{ij}}}{\mathrm{r}_{\mathrm{o}}},
$$

where $\mathrm{V}$ and $\mathrm{r}_{\mathrm{ij}}$ are respectively the energy and the interatomic distance in absolute units, $\mathrm{r}_{\mathrm{o}}$ is fixed at the equilibrium value of the bulk nearest-neighbor distance in absolute units, and $U$ can be viewed as a unit of energy. (It has been suggested to consider $r_{0}$ and $U$ as parameters that depend not only on the metal but also on the cluster size [31-34]. For calibration one can use the values $\mathrm{U}=3.77 \mathrm{eV}$ for nickel and $\mathrm{U}=3.69 \mathrm{eV}$ for gold, which are calculated from the bulk cohesive energies of the two fcc metals [32]). The value of the parameter A is obtained from the condition that the fcc lattice arrangement of atoms, with a lattice constant defined by the nearest-neighbor distance $r_{0}$, maximizes the cohesive energy of the bulk metal. The values of $\mathrm{A}$ for nickel and gold are 0.101036 and 0.118438 , respectively [31-34]. For a system of identical atoms of mass $m$ one can express time $t$ in units of $\tau_{0}, t^{*}=t / \tau_{0}$, where $\tau_{0}$ is defined as 


$$
\tau_{0}=r_{0} \sqrt{\frac{m}{U}}
$$

Newton's equations of motion (5') then take the form

$$
\frac{d^{2} r_{i}^{*}}{d t^{* 2}}=-\frac{\partial V^{*}}{\partial r_{i}^{*}}
$$

where $r_{i}^{*}=r_{i} / r_{0}$.

Intracluster dynamics corresponding to fixed values of the total energy are obtained by solving numerically Eqs. (3), (5') or (29). Hamming's modified predictor-corrector algorithm [35] is used to propagate Hamilton's equations, whereas the Verlet integrator [36] is applied to Newton's equations. The initial coordinates and momenta (velocities) of the atoms are chosen to supply zero total linear and angular momenta to a cluster. The lengths of the individual trajectories are of the order of $10^{5}-10^{6}$ steps. The propagation step sizes used are of the order of $10^{-15} \mathrm{~s}$. The total energy is conserved in the individual runs typically within a few hundredths of a percent.

The $\mathrm{Ni}_{13}+\mathrm{D}_{2}$ system is considered below as a paradigm of cluster-ligand interactions. $\mathrm{Ni}_{13}$ is modeled by Voter and Chen's embedded-atom potential [27] discussed above. The $\mathrm{D}-\mathrm{D}$ and $\mathrm{D}_{2}-\mathrm{Ni}_{13}$ interactions are mimicked by a London-Eyring-Polanyi-Sato (LEPS) potential energy surface (PES) VLEPS. Apart from a minor modification by a smoothing function of the form utilized by Truong et al. [37], the PESII of Raghavan et al. [38] is used to represent $V_{\text {LEPS }}$. Results on interaction of $\mathrm{D}_{2}$ with $\mathrm{Ni}_{13}$ are derived from quasiclassical trajectory simulations. The initial coordinates and momenta of the $\mathrm{D}$ atoms are chosen to supply the $\mathrm{D}_{2}$ molecule an amount of energy that corresponds to specified vibrational $v$ and rotational $j$ quantum numbers. This is accomplished using the quasiclassical quantization procedure described in [39]. The COM of the molecule is placed initially "asymptotically far" (8.5 $\AA$ ) from the COM of the cluster. The molecule is then sent toward the cluster with a specified relative translational (collision) energy $E_{t r}$ and impact parameter $b$. The initial coordinates and momenta of the atoms of the nontranslating and nonrotating $\mathrm{Ni}_{13}$ are chosen to correspond to a given structure and/or temperature of the cluster. Phase space trajectories representing the time evolution of the clustermolecule collision system are generated by solving numerically Hamilton's equations of motion for all the fifteen atoms. Hamming's propagator with a maximal step size of $5 \cdot 10^{-16} \mathrm{~s}$ is used for this. The individual trajectories conserve the total energy of the cluster-molecule system within $0.03 \%$. Each trajectory is run until either of the two possible eventualities takes place: a) the molecule dissociates on the cluster, in which case the trajectory is qualified as a reactive one; b) after collision with the cluster the molecule departs back into the asymptotic region, which is the case of a nonreactive trajectory. The molecule is viewed as dissociated when and if the distance between the D atoms 
becomes equal to $3 d_{0}$, where $d_{0}=0.741 \AA$ is the equilibrium bond length of $D_{2}$. Results on the reaction (i.e., dissociative adsorption of the molecule on the cluster) derived from the simulations are presented and discussed below. Comparison with the available experimental data is used to assess the degree of adequacy of the PESII parameterization of the $\mathrm{Ni}_{13}-\mathrm{D}_{2}$ interaction potential. Being guided by the measured value of the energy of binding of a $\mathrm{D}$ atom to a surface of bulk nickel, we perform a fine "tuning" of this potential. Details of the tuning and its consequences are discussed in Section 6.

\section{Cluster Structures}

The structures of clusters are obtained through simulated thermal quenching [31-34,40,41]. High energy points in the phase space of a cluster are used as initial conditions for the runs. The runs are intermittently stopped and the momenta of all the atoms are put equal to zero. This process is continued until a cluster freezes into one of its minimum energy structures (isomeric forms). The most stable geometric forms of $\mathrm{Ni}_{n}, \mathrm{n}=2-14$, as obtained from the Gupta-like potential, are shown in Fig. 1. The embedded-atom potential $V_{E A}$ leads to the same lowest energy structures. Figure 2 displays the most stable isomers of $\mathrm{Ni}_{\mathbf{n}}$, $\mathrm{n}=9,12-14,15,18-20,55$, as defined by $\mathrm{V}_{\mathrm{EA}}$. One notices the recurring pattern of icosahedral (ico) packing. $\mathrm{Ni}_{13}$ and $\mathrm{Ni}_{55}$ are complete icosahedra, $\mathrm{Ni}_{19}$ is a socalled double icosahedron, whereas $\mathrm{Ni}_{\mathrm{n}}, \mathrm{n}=9-12,14,15,18,20$, are icosahedra with either missing or extra decorating atoms. Most minimum energy structures of small nickel clusters shown in Figs. 1 and 2 agree with those obtained using alternative theoretical approaches $[38,42]$ or inferred from experiments [43].

A characteristic feature of clusters is that for a fixed size they form (usually) a large number of isomeric structures. Each isomer corresponds to a global or local minimum of the corresponding $(3 n-6)$-dimensional potential energy surface. Five structures of Nig obtained from $V_{E A}$ are shown in Fig. 3. Some of these structures have very close energies. Figure 4 displays three highly symmetrical forms of $\mathrm{Ni}_{13}$. The energies of the hexagonal closed-packed (hcp) and cuboctahedral (cubo) forms, as obtained from $V_{E A}$, are again very close. The lowest energy structure of $\mathrm{Ni}_{14}$ is a slightly relaxed 13-atom ico with an extra atom in a threefold hollow site (i.e., over a face); cf. Fig. 2. Another topologically similar isomer of this cluster, which is $0.19 \mathrm{eV}$ higher in energy, is obtained by placing the fourteenth atom in a twofold site (i.e., over an edge) of a slightly relaxed ico $\mathrm{Ni}_{13}$. Three isomers of $\mathrm{Ni}_{55}$ obtained from $\mathrm{V}_{\mathrm{EA}}$ are shown in Fig. 5. Especially interesting is the second isomer labeled as "defico". It can be identified as a 55-atom ico with a defect. Instead of a missing edge atom, an extra atom appears on the opposite side of the cluster causing a small relaxation away from the ideal ico geometry. Figure 6 displays isomeric forms of $\mathrm{Au}_{12}$ and $\mathrm{Au}_{14}$ obtained from the Gupta-like potential. In distinct difference to $\mathrm{Ni}_{12}$ and $\mathrm{Ni}_{14}$, the most stable structures of $\mathrm{Au}_{12}$ and $\mathrm{Au}_{14}$ are not ico-based, albeit $\mathrm{Au}_{13}$ (not shown) is a complete icosahedron. A plausible explanation for this disparity in the structures of nickel and gold clusters is that the ranges and the relative contributions of the attractive and repulsive 


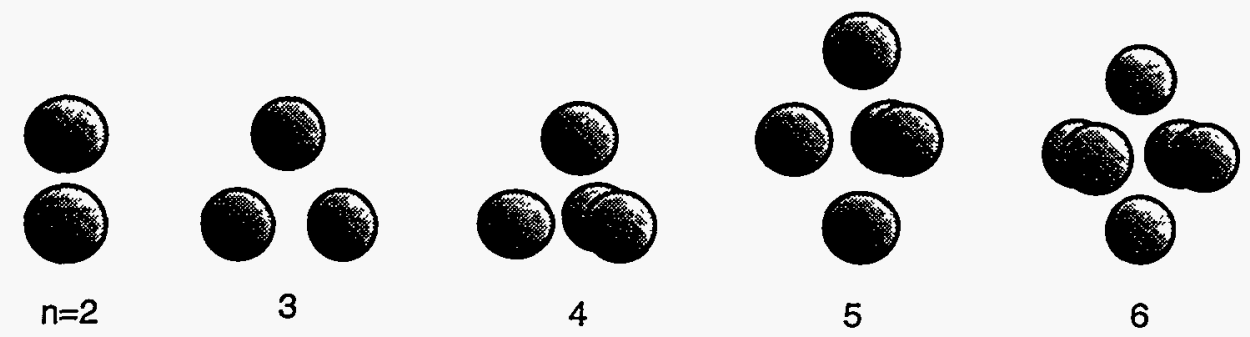

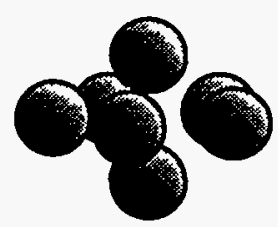

7

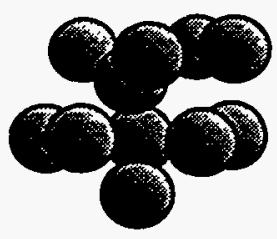

11

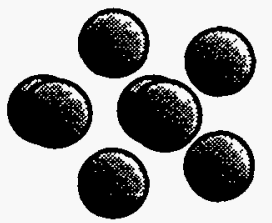

8

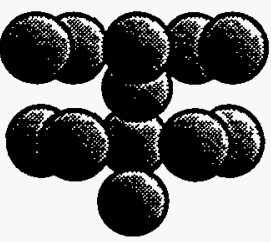

12

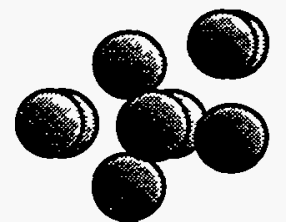

9

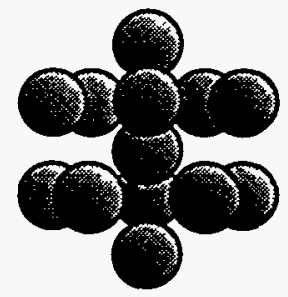

13

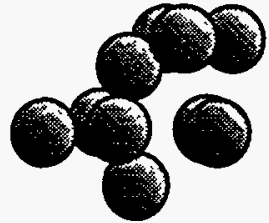

10

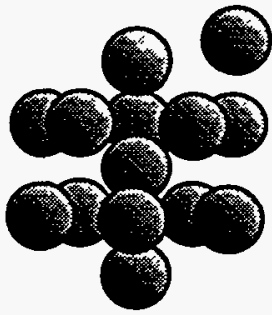

14

Figure 1. The most stable structures of $\mathrm{Ni}_{\mathrm{n}}, \mathrm{n}=2-14$, clusters as obtained from the Gupta-like potential. The energies of these clusters in reduced units are: -1.211 , $-2.160,-3.187,-4.170,-5.215,-6.209,-7.193,-8.200,-9.222,-10.234,-11.292$, -12.463 , and -13.398 , respectively.

interactions in the two metals are different. With the possible exception of the most stable structure, the isomers of $\mathrm{Au}_{14}$ are more spherical than those of $\mathrm{Au}_{12}$. Examination of Fig. 6 indicates again that isomers of a cluster may have close energies even when their geometries are quite different.

As mentioned, bulk nickel and gold are fcc metals. However, the cubo isomers of small nickel and gold clusters, which can be viewed as fragments of the fcc lattice, are not the energetically most preferred structural forms of these clusters (cf., e.g., Figs. 4 and 5). Studies of clusters of other metals, as well as nonmetal materials, indicate that the most favorable geometries in the small [tens of atoms (molecules)] and intermediate [hundreds to a few thousands of atoms (molecules)] size ranges are usually different from the corresponding bulk structures. An important question then is: What are the smallest sizes at which lattices become the energetically most preferred arrangements, and how do these sizes depend on the nature of the interatomic forces [44-46]? A related 

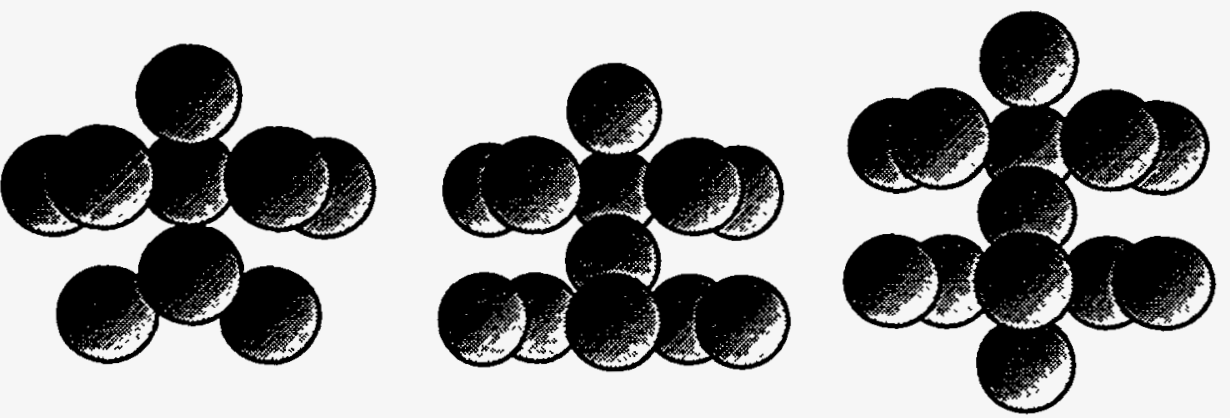

$n=9$

12

13
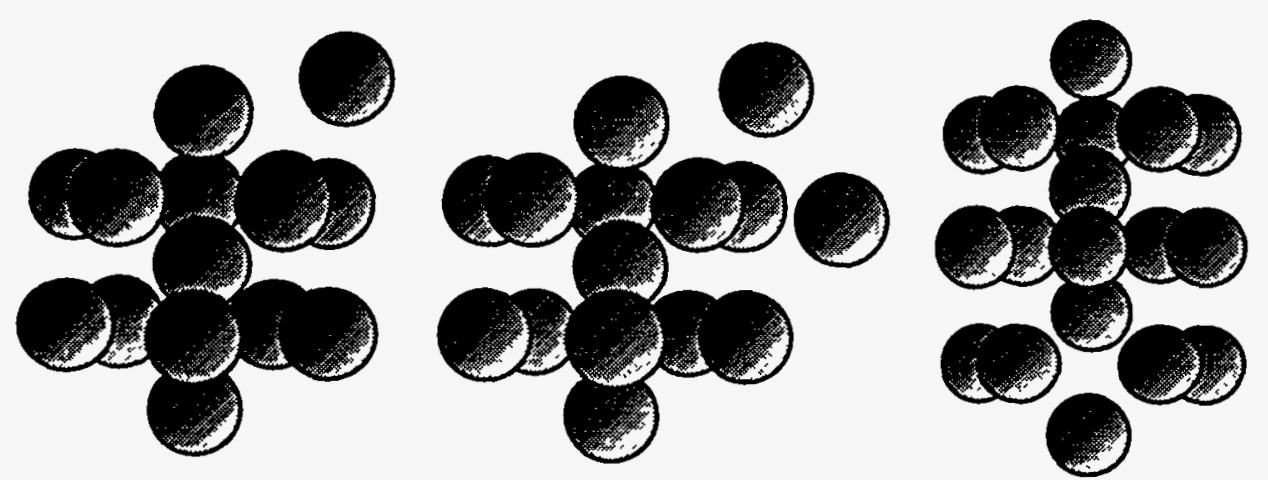

14

15

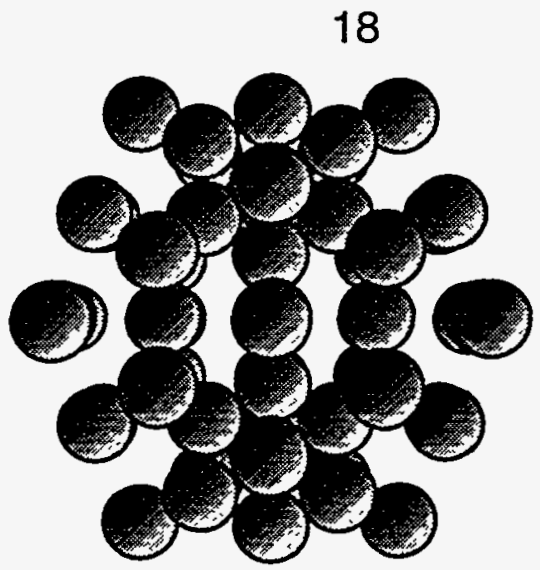

19

20

55

Figure 2. Most stable structures of $\mathrm{Ni}_{\mathrm{n}}$ clusters as obtained from $\mathrm{V}_{\mathrm{EA}}$. Their energies in $\mathrm{eV}$ are: $n=9:-25.29 ; n=12:-36.48 ; n=13:-41.12 ; n=14:-44.09 ; n=15:-47.70 ;$ $n=18:-58.52 ; n=19:-63.01 ; n=20:-65.98 ; n=55:-203.02$. 


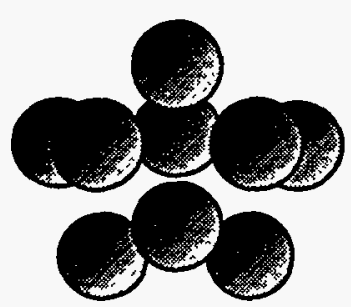

Isomer I

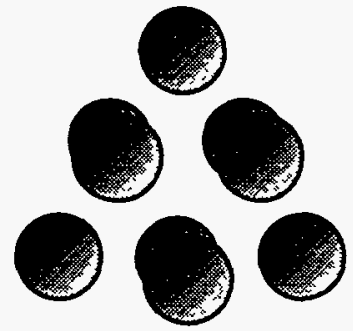

II

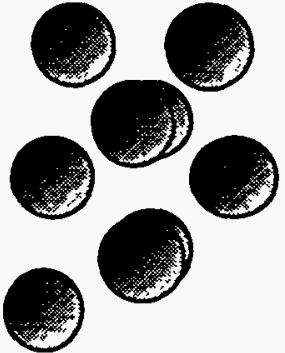

III

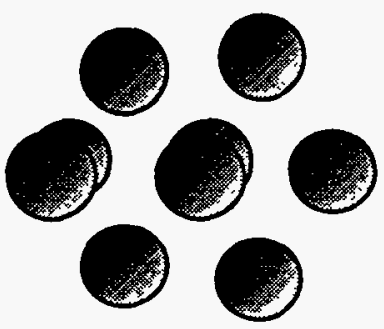

IV

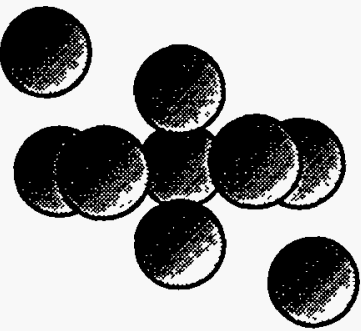

V

Figure 3. Isomers of $\mathrm{Nig}$ as obtained from $\mathrm{V}_{\mathrm{EA}}$. Their energies in $\mathrm{eV}$ are: I: -25.29 ; II: -25.20 ; III: -24.86 ; IV: -24.84 ; V: -24.65 .

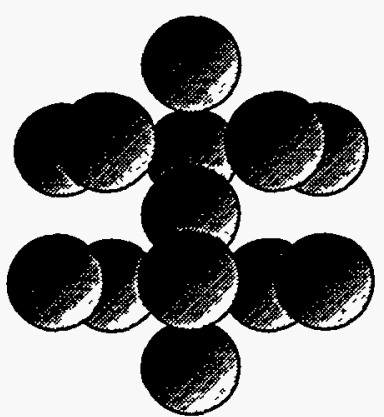

ico

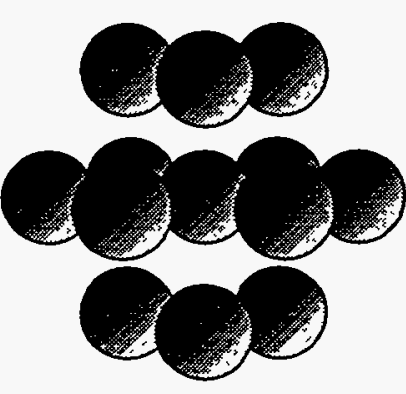

hcp

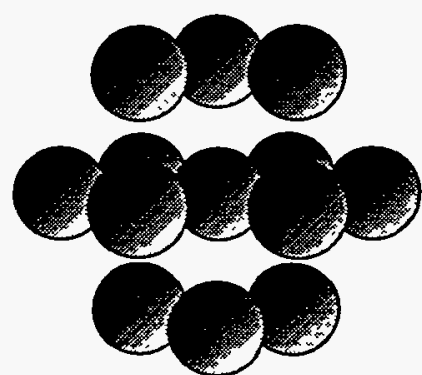

cubo

Figure 4. Highly symmetrical structures of $\mathrm{Ni}_{13}$ obtained from $\mathrm{V}_{E A}$. Their energies in $\mathrm{VV}$ are: ico: -41.12 ; hcp: -39.53 ; cubo: -39.51 . 

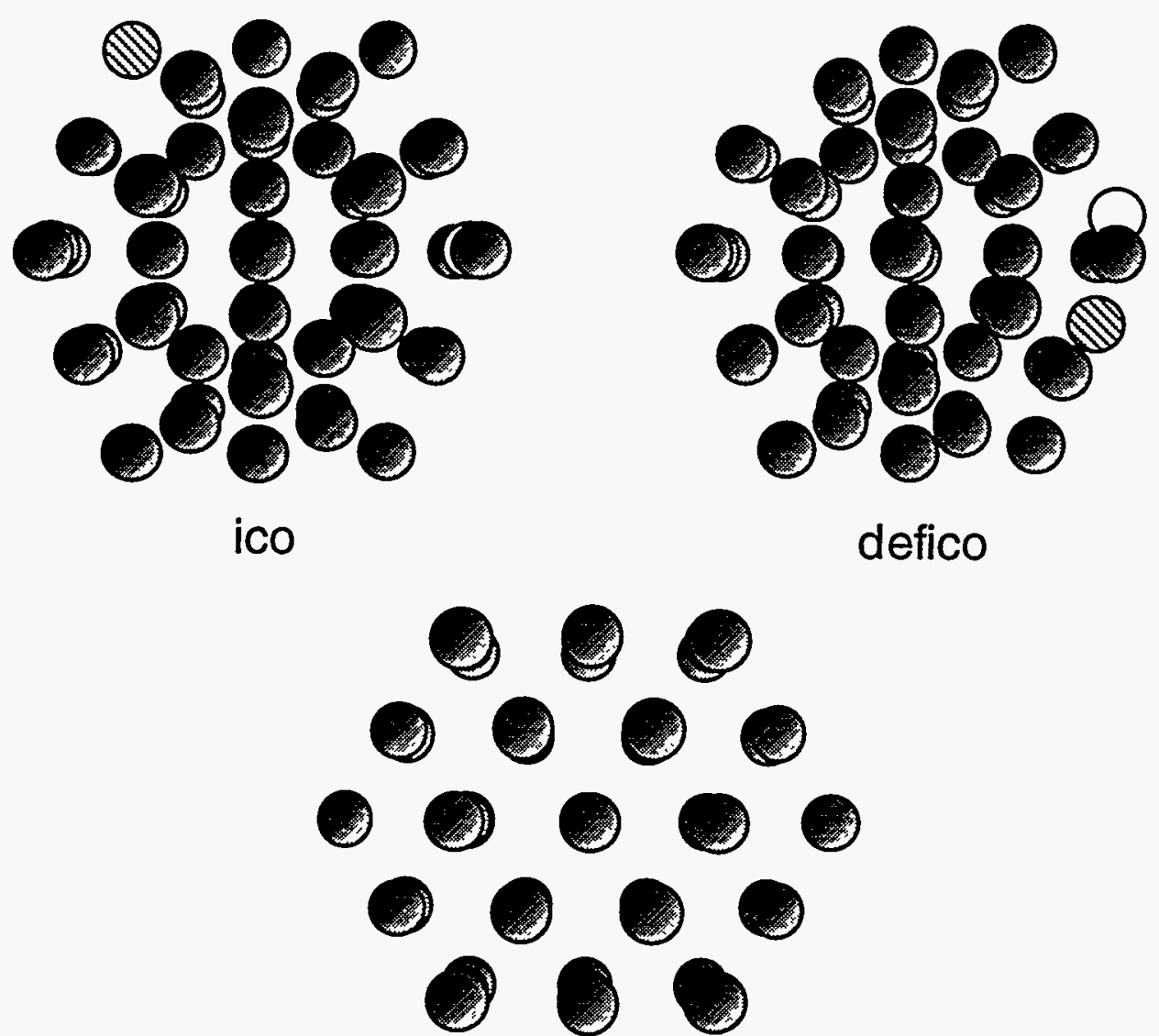

cubo

Figure 5. Isomers of $\mathrm{Ni}_{55}$ obtained from $\mathrm{V}_{\mathrm{EA}}$. Their energies in $\mathrm{eV}$ are: ico: -203.02; defico: -201.68; cubo: -199.63 . The shade-coding is used to establish a correspondence between the structures rather than between the individual atoms of the ico and defico isomers.

question refers to the evolution of cluster geometries with their size and the pathways for conversion of these geometries into bulk structures. There are indications that the pathways may be quite complex in that they may involve different intermediate polycrystalline states [47].

\section{Intracluster Dynamics: Isomerization and Melting}

In this section we give an analysis of the intracluster dynamics as defined by cluster size and internal energy, or temperature [31-34,40,48-50]. Features of the dynamics are derived from constant-energy $M D$ simulations, in which the temperature $\mathrm{T}$ is defined as 


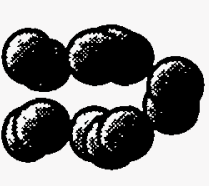

Isomer I

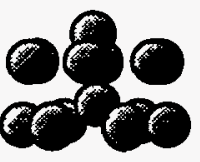

I

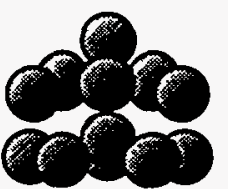

II

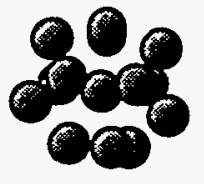

II

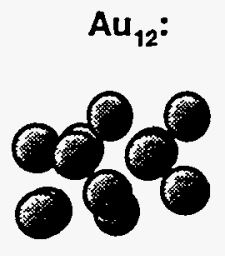

III

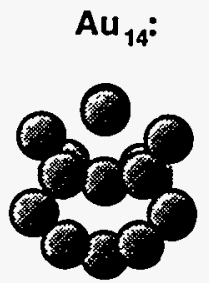

III

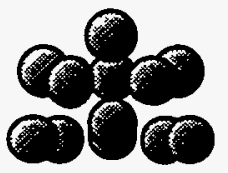

IV

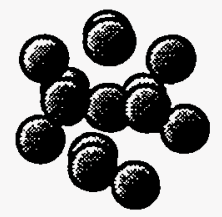

IV

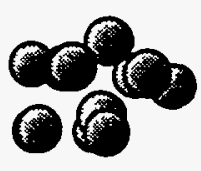

V

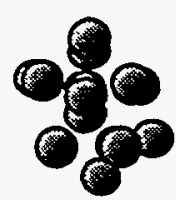

V

Figure 6. Isomers of $\mathrm{Au}_{12}$ and $\mathrm{Au}_{14}$ obtained from the Gupta-like potential. Their energies in reduced units are: $\mathrm{Au}_{12}$ : I: -10.804 ; II: $-10.790 ; \mathrm{III}:-10.788$; IV: -10.769 ;

$\mathrm{V}:-10.761 ; \mathrm{Au}_{14}$ : I: -12.745; II: -12.711; III: -12.705; IV: $-12.699 ; \mathrm{V}:-12.688$.

$$
T=\frac{\left.2<E_{k}\right\rangle}{(3 n-6) k}
$$

where $E_{k}$ is the total internal kinetic energy of a cluster, $k$ is the Boltzmann constant, and $<>$ denotes time-averaging over a long trajectory. Features of the dynamics depend, of course, on the nature of the interatomic interactions. Clusters of different materials, however, show similar qualitative changes in their dynamics as their internal energy is increased. The common elements include isomerization and meltinglike transitions. Bulk melting, which in crystalline materials is a first order phase transition, is usually viewed and treated as a collective thermodynamic phenomenon. It can, however, be understood also in dynamical terms as a transition from a restricted and correlated (periodic) motion of atoms, characteristic of solids, to an essentially uncorrelated motion, characteristic of liquids. A detailed dynamical description of bulk melting is impractical because of too large a number of atoms (degrees of freedom) to monitor. A dynamical analysis of the meltinglike transition in small and intermediate size clusters is feasible. It allows one to extract and to characterize the peculiarities of the analog of bulk melting in finite systems.

The common elements in the evolution of cluster dynamics with energy can be understood in terms of topology of the multidimensional potential energy surfaces. (As specified above, we consider here nonrotating and nontranslating 
clusters. Therefore the total energy of a cluster is its internal energy. We can abstract ourselves from the translational motion since it has no effect on the meltinglike phenomenon. In general, this is not true for the rotational motion. The available energy may be partitioned between the vibrational modes and a possible overall rotation. For an analysis of the effects of the rotational motion on cluster dynamics see $[15,18])$. At low energies the trajectories representing the dynamical evolution of a cluster are confined to a part of its configuration space ("basin of attraction", "catchment area") that corresponds only to one isomer. The identity of this isomer is defined by the initial values of the coordinates. One usually (but not necessarily, cf. [11]) starts from a configuration that derives from the most stable structure of a cluster. As the cluster energy increases and exceeds the value of the lowest potential energy barrier (saddle) separating the catchment area of the initial isomeric form, the dynamics find their way over this barrier into the basin(s) of attraction of another isomer(s). To observe an interbasin (isomerization) transition one may have to wait for quite some time. This time is spent on channeling the energy into the collective "reaction" (in this case, "isomerization") coordinate. (A measure zero manifold of initial conditions, which specify so-called "trapped" trajectories, will not lead to a transition even when it is energetically allowed). With further increase of the energy the number of different catchment areas (isomeric forms) sampled by the dynamics increases. But the interbasin transitions remain separated by "dwell" times that are sufficiently long, as measured by the characteristic vibrational period of the cluster. During the dwell times the dynamics remain confined to the individual catchment areas and, therefore, the isomeric forms of a cluster can be identified. This pattern of cluster dynamics can be labeled as corresponding to the isomerization or, alternatively, "coexistence" stage. The coexistence stage in cluster melting was first predicted from an analytical model [51]. An important aspect of the prediction is that the coexistence stage corresponds to a finite range of energy (or temperature), which can be called the coexistence range. The finite size of the coexistence range has been confirmed in numerical simulations of clusters of many materials. The origin of the term "coexistence" is in the ensemble interpretation of the phenomenon. In a microcanonical (canonical) ensemble of clusters characterized by a fixed energy (temperature) from the coexistence range one finds that different isomeric forms of a cluster coexist. The dynamical meaning of the term is that different isomers of a cluster are sampled, and in this sense (sequentially) coexist, along a single trajectory that corresponds to an energy (temperature) from the coexistence range.

As the energy (temperature) in the coexistence range increases, the cluster dynamics sample larger parts of the configuration space, the dwell times decrease, and the rate of isomerization transitions increases. Eventually the dwell times become equal to or even smaller than the characteristic vibrational period of the cluster and no individual isomeric forms survive long enough to be identified. The characteristic correlation time and length become very small the cluster acquires a liquidlike state. (We exclude from consideration the 
fragmentation phenomenon, which may become feasible at or slightly above energies necessary to melt a cluster; $c f .[41])$.

Numerical simulations $[2,31-34,40,48-50]$ confirm that the general picture of the dynamics of solid-to-liquidlike transitions presented above applies to clusters of various materials, including metals. These simulations, however, also provide evidence for new elements (stages) in the meltinglike transition of metal clusters. A variety of characteristic quantities can be used to analyze cluster melting $[49,50]$. These include:

1) Short-time averaged (internal) kinetic energy (per atom) $\overrightarrow{\mathrm{E}}_{\mathrm{k}}$ as a function of time. The averaging is performed over intervals of $5 \cdot 10^{-13} \mathrm{~s}$, which correspond to approximately five characteristic vibrational periods. (The vibrational period of $\mathrm{Ni}_{2}$ is $1.01 \cdot 10^{-13} \mathrm{~s}$ and the period corresponding to the Debye frequency of bulk nickel is $1.18 \cdot 10^{-13} \mathrm{~s}$.) The averaging is performed to suppress the variations in the internal kinetic energy due to vibrational motion of the atoms;

2) Relative root-mean-square (rms) bond length fluctuation $\delta$,

$$
\delta=\frac{2}{n(n-1)} \sum_{i, j(>i)=1}^{n} \frac{\left.\left(<r_{i j}^{2}>-<r_{i j}\right\rangle^{2}\right)^{1 / 2}}{<r_{i j}>}
$$

3) Mean square displacement $\Delta \mathbf{r}^{2}(\mathrm{t})$,

$$
\Delta r^{2}(t)=\frac{1}{n \cdot n_{t}} \sum_{j=1}^{n_{t}} \sum_{i=1}^{n}\left[r_{i}\left(t_{o j}+t\right)-r_{i}\left(t_{o j}\right)\right]^{2}
$$

where $n_{t}$ is the number of different time origins $t_{0 j}$;

4) Diffusion coefficient $D$,

$$
D=\frac{1}{6} \lim _{t \rightarrow \infty} \frac{d\left(\Delta r^{2}(t)\right)}{d t}
$$

Here we present results for nickel clusters derived from $V_{E A}$. Figure 7 displays patterns of $\overline{\mathrm{E}}_{\mathrm{k}}(\mathrm{t})$ for $\mathrm{Ni}_{13}$ at different energies of the cluster. Because variations in the kinetic energy due to vibrations are suppressed and the total energy is conserved along a trajectory, near constancy of $\bar{E}_{k}^{\prime}(t)$ means near constancy of the short-time averaged potential energy of the cluster. Therefore, barring cases of energetic degeneracy of different isomers, near constancy of $\overline{\mathrm{E}}_{\mathrm{k}}^{\prime}(\mathrm{t})$ means that the dynamics are confined to the catchment area of only one structural form of the cluster. At low energies corresponding to graphs a) and b) the dynamics of $\mathrm{Ni}_{13}$ explore the basin of attraction of its most stable ico isomer. This is confirmed through many thermal quenchings from configurations recorded along the trajectories that generate graphs a) and b). In this sense the cluster is solidlike at energies corresponding to graphs a) and b). At higher energy corresponding to graph $c$ ) the cluster explores different 


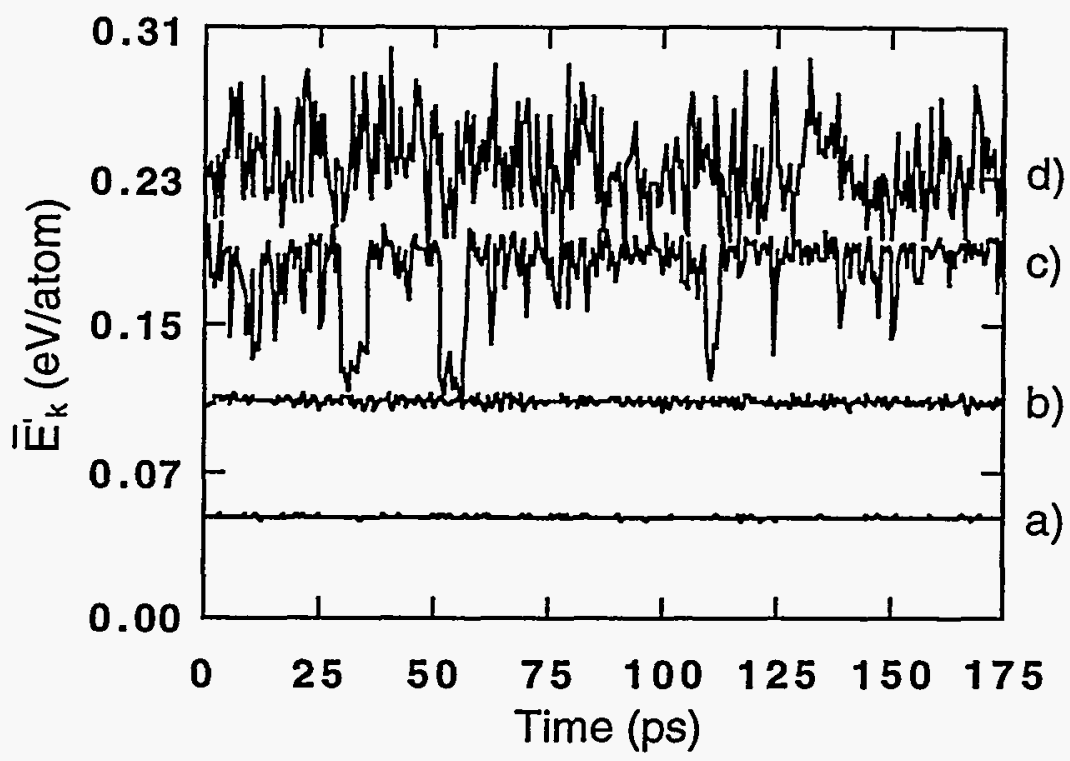

Figure 7. Short-time averaged kinetic energy per atom as a function of time for $\mathrm{Ni}_{13}$. The graphs correspond to the following total energies (in $\mathrm{eV}$ ) per atom: a) -3.05 ;

b) -2.92 ; c) -2.73 ; d) -2.49 .

isomeric structures. Each structure is represented by a well-identifiable branch of the graph. The individual branches persist for appreciable times and are revisited in the course of the simulation run. The highest- $\bar{E}_{\mathrm{k}}^{\prime}$ branch corresponds to the lowest potential energy ico isomer. The lower- $\vec{E}_{k}^{\prime}$ branches represent metastable isomers of higher potential energy. The pattern of graph $c$ ) is typical of the coexistence stage. The behavior of $\overline{\mathrm{E}}_{\mathrm{k}}(\mathrm{t})$ at still higher energy is represented by graph d). Large fluctuations and the almost complete disappearance of resolvable individual branches are consequences of frequent interbasin excursions, which signify that the cluster became liquidlike.

Graphs of the mean square displacement for two energies considered in Fig. 7 are shown in Fig. 8 (the labeling of the graphs corresponds to that in Fig. 7). The graphs corroborate the assignment of the solidlike and liquidlike states of the cluster. The zero slope of the long-time tale of graph b) indicates no diffusion of the atoms, as it should be in a solidlike state [cf. Eq. (33)]. The large slope of graph d) signifies a well-developed diffusional motion, which is typical of a liquidlike state. Yet another corroboration of the meltinglike transition in $\mathrm{Ni}_{13}$ is presented in Fig. 9, which displays the rms bond length fluctuation $\delta$ as a function of the cluster temperature. The abrupt change in the values of $\delta$ is analogous to the Lindemann criterion of bulk melting [52]. A feature to note is that the transition starts only when the temperature of the cluster reaches the value of $\approx 1300 \mathrm{~K}$, which indicates a high degree of stability of $\mathrm{Ni}_{13}$. (The melting temperature of bulk nickel is $1726 \mathrm{~K}$.) The general qualitative features of the solid-to-liquidlike transition in $\mathrm{Ni}_{13}$, with the possible 


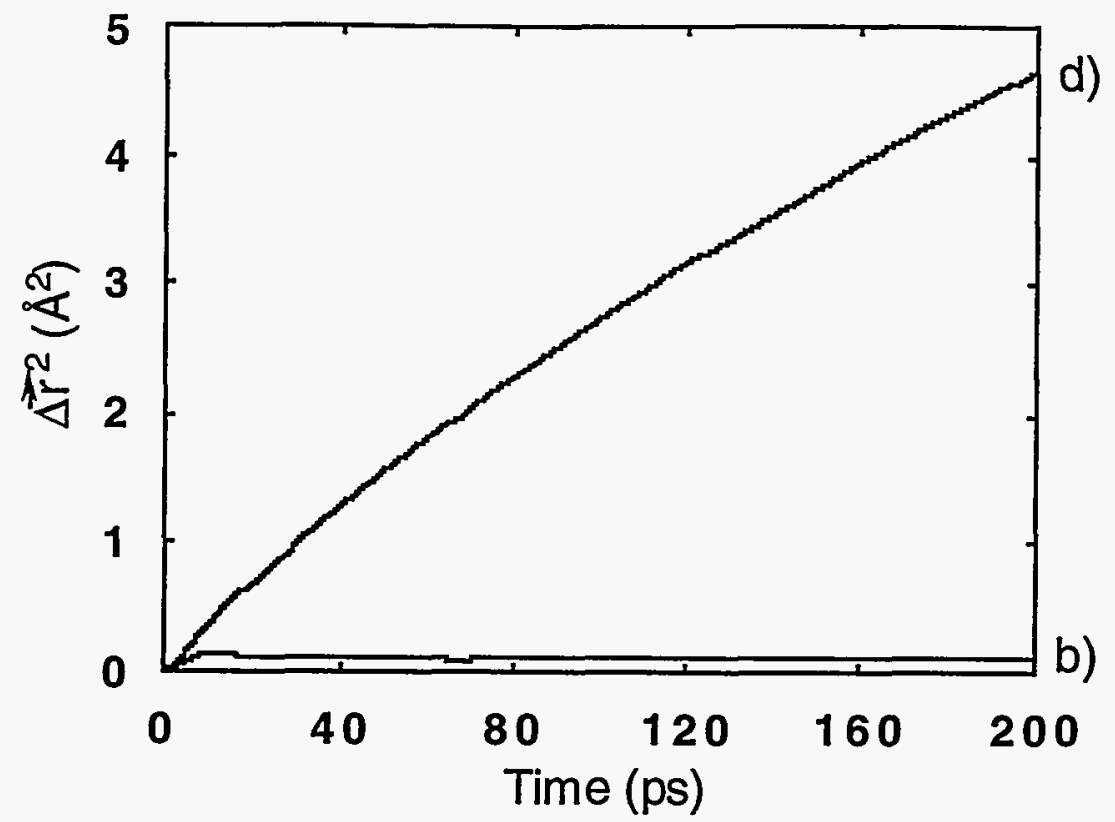

Figure 8. Mean square displacement as a function of time for $\mathrm{Ni}_{13}$. Graphs b) and d) correspond respectively to the same total energies as graphs b) and d) of Fig. 7.

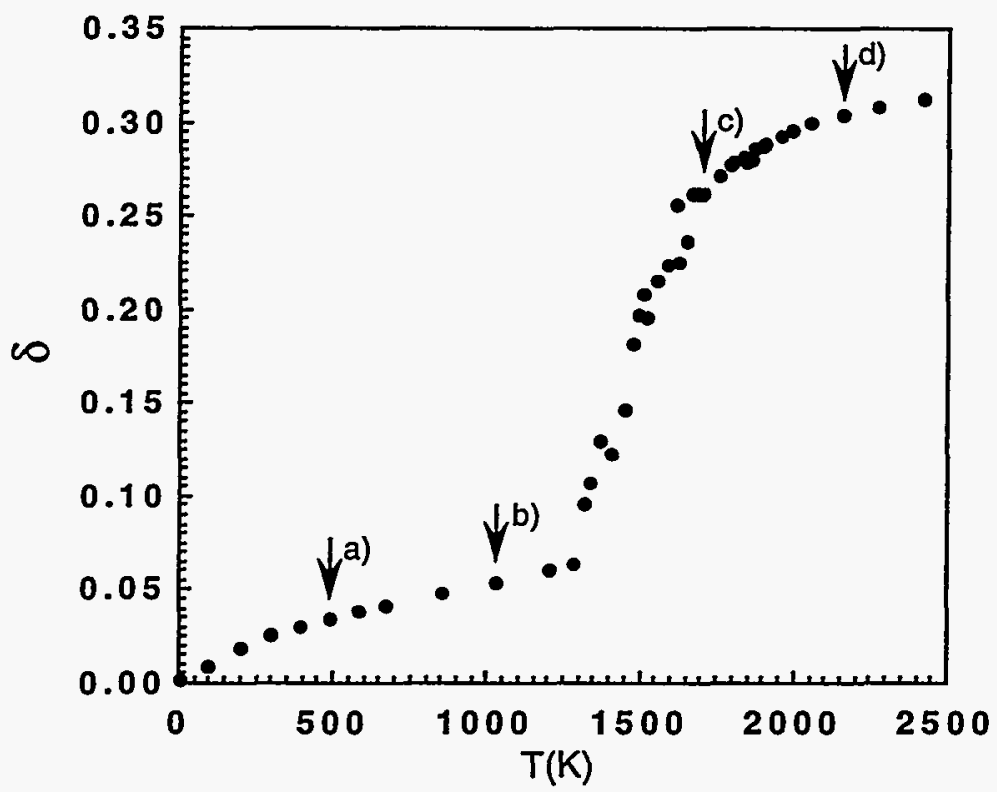

Figure 9. Relative rms bond length fluctuation as a function of cluster temperature for $\mathrm{Ni}_{13}$. Arrows indicate points of the graph that correspond to the total energies considered in Fig. 7. 
exception of its extra stability, are shared by nickel clusters of other sizes, such as, for example, $\mathrm{Ni}_{12}, \mathrm{Ni}_{15}, \mathrm{Ni}_{18}, \mathrm{Ni}_{19}$, etc. The double-icosahedral $\mathrm{Ni}_{19}$ (Fig. 2) also shows a considerable resistivity to melting.

But not all nickel clusters behave like $\mathrm{Ni}_{13}$. Figure 10 shows the graph of the rms bond length fluctuation $\delta$ for $\mathrm{Ni}_{14}$. Instead of one, there are two abrupt changes in the values of $\delta$. Such a pattern of $\delta$ has not been obtained for nonmetallic clusters. We have used computer visualization to uncover the intricate details in the meltinglike transition of this cluster. The first abrupt change in $\delta$, which starts at $T \approx 300 \mathrm{~K}$, corresponds to the so-called premelting phenomenon [31-40]. This phenomenon involves only the two lowest energy ico-based isomers of $\mathrm{Ni}_{14}$ described above. Premelting involves coexistence of these isomers, i.e., occasional migration of the fourteenth atom from a threefold site to a twofold site and back, which is accompanied only by a small adjustment of the underlying ico 13-mer. In the next stage of premelting, represented by the approximately horizontal part of the graph, the frequency of the isomerization transitions increases and the fourteenth atom becomes capable of inserting itself into the framework of the 13-mer. This insertion leads to expulsion of a neighboring atom, which becomes the "extra" fourteenth atom. The motion of the newly formed fourteenth atom continues to generate the aforementioned frequent isomerizations and leads to new insertion-expulsion processes. However, only those atoms of the cluster that are neighbors of the instantaneous fourteenth atom participate in large amplitude motions. The part of the 13-mer that is not adjacent to the fourteenth atom largely preserves its

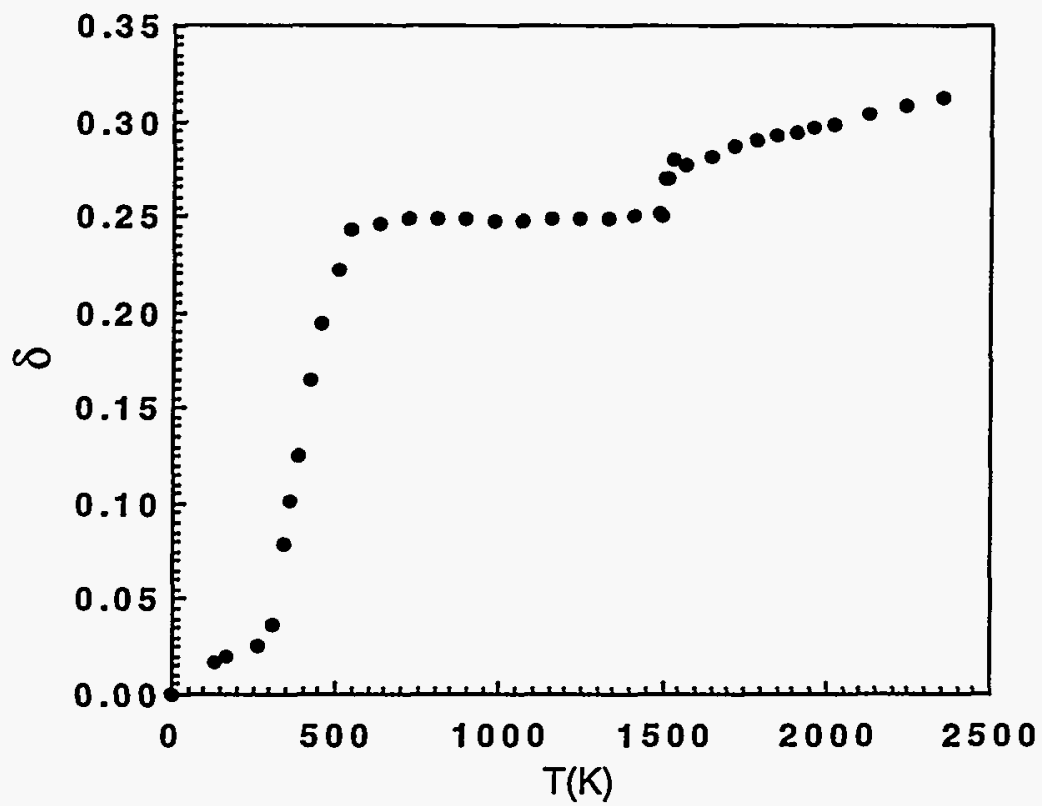

Figure 10. Relative rms bond length fluctuation as a function of cluster temperature for $\mathrm{Ni}_{14}$. 
icosahedral packing. The essence of the premelting phenomenon is local melting caused by the destabilizing effect of the fourteenth atom on the adjacent part of the otherwise very stable ico 13-mer. The second abrupt change in $\delta$ values, which takes place at considerably higher temperatures, corresponds to the melting of the entire $\mathrm{Ni}_{14}$. This requires melting the underlying 13-mer. The analysis shows that all the individual stages described above for $\mathrm{Ni}_{13}$ can be identified. Inspection of Figs. 9 and 10 confirms that the complete melting of $\mathrm{Ni}_{14}$ takes place essentially over the same temperature range as that of $\mathrm{Ni}_{13}$. Another cluster that exhibits the premelting stage followed by a complete melting is $\mathrm{Ni}_{20}$ [40]. $\mathrm{Ni}_{20}$ is similar to $\mathrm{Ni}_{14}$ in that it is also formed by a stable network (the double icosahedral 19-mer) and an additional atom in a threefold hollow site (Fig. 2). The destabilizing effect exerted by the twentieth atom explains the premelting phenomenon in this cluster. We conjecture that the premelting (local melting) stage will be part of the meltinglike transition in small nickel clusters of those sizes that form stable (e.g., icosahedral) structures with one extra surface atom. We obtained the premelting phenomenon in $\mathrm{Ni}_{14}$ and $\mathrm{Ni}_{20}$ from both the $\mathrm{V}_{\mathrm{EA}}$ and the Gupta-like potential. The many-body character of these potentials plays an important role in defining premelting. This, however, does not mean that every 14- or 20-atom cluster described by a many-body potential exhibits premelting. $\mathrm{Au}_{14}$, for example, modeled by the Gupta-like potential does not support local melting [34]. The reason for this is that the most stable structure of this cluster (Fig. 6), which is different from that of $\mathrm{Ni}_{14}$, does not involve a highly stable underlying 13-atom network.

A 55-atom ico cluster is the smallest one with two complete spherical shells. The inner part, or the core, of such a cluster is a 13-atom icosahedron. The response of an ico $\mathrm{Ni}_{55}$, which, as mentioned above (Figs. 2 and 5), is the most stable isomer of this cluster, to an increase of its temperature is analyzed in terms of the rms bond length fluctuation $\delta$ in Fig. 11. The three graphs correspond to $\delta$ values calculated separately for the 13-atom core, 42-atom surface layer, and the entire $\mathrm{Ni}_{55}$. The partitioning into core and surface atoms is meaningful up to $T \approx 1100 \mathrm{~K}$. Above this temperature the atoms migrate between the surface and the core with increasing frequency. At $\mathrm{T} \leq 830 \mathrm{~K}$ the values of the three $\delta$ 's are small and essentially equal - the cluster is solidlike. In the range $T \approx 830-1100 \mathrm{~K}$ the values of $\delta$ for surface atoms are larger and grow faster than those for core atoms. The surface of the cluster becomes liquidlike, while its core remains solidlike. Thus melting of $\mathrm{Ni}_{55}$ involves the stage of surface melting characterized by a finite temperature range, in which the cluster can be viewed as a liquidlike mantle enveloping a solidlike core. At temperatures slightly above $1100 \mathrm{~K}$ the $\delta$ values for the core increase abruptly and the three graphs merge. The large values of the $\delta$ 's and the indistinguishability between the core and surface atoms indicate that the entire cluster becomes liquidlike. Complementary data are presented in Table 1, which displays the diffusion coefficients for the three groups of atoms at different temperatures (further details are given in [48]). Surface melting has also been predicted for $\mathrm{Cu}_{55}$ and noble gas clusters of a variety of sizes [53], as well as gold clusters of larger sizes [54]. 


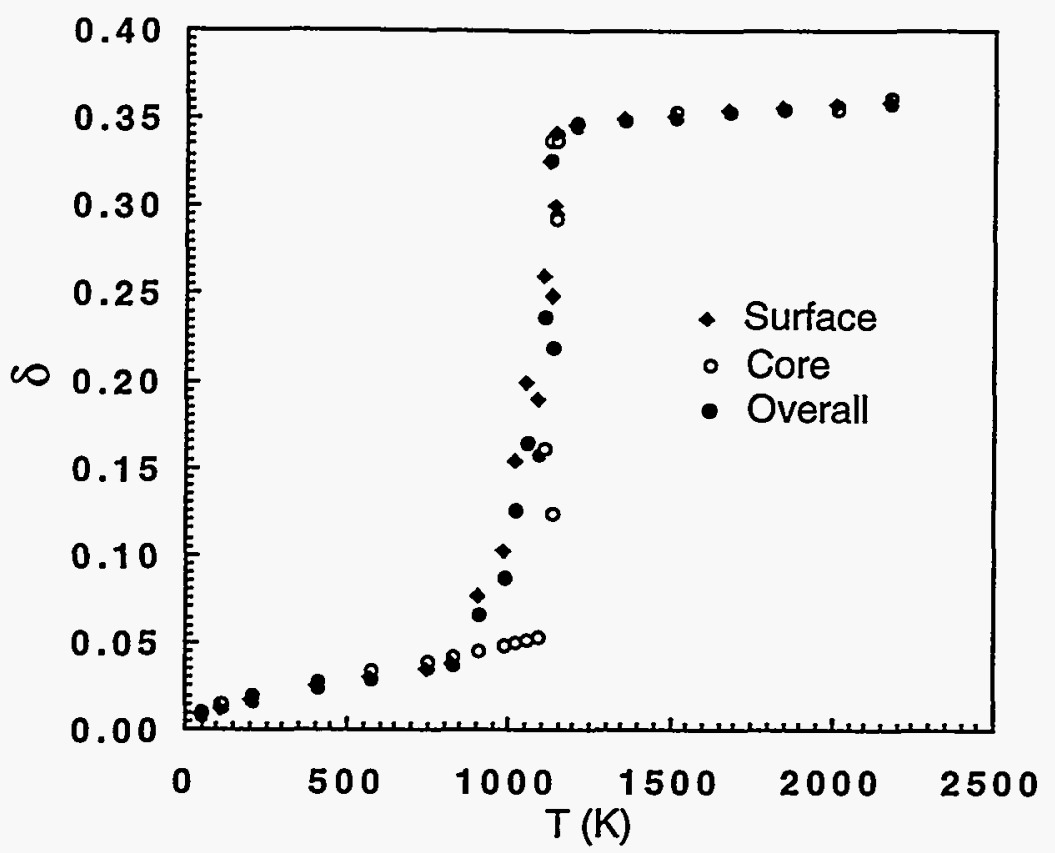

Figure 11. Relative rms bond length fluctuation as a function of cluster temperature for $\mathrm{Ni}_{55}$ (see text).

TABLE 1. Diffusion coefficients (in $\mathrm{cm}^{2} / \mathrm{s}$ ) as a function of temperature for $\mathrm{Ni}_{55}$.

\begin{tabular}{cccc}
\hline T(K) & CORE & SURFACE & OVERALL \\
\hline 581 & $1.17 \times 10^{-9}$ & $4.67 \times 10^{-9}$ & $3.83 \times 10^{-9}$ \\
751 & $1.07 \times 10^{-9}$ & $5.53 \times 10^{-9}$ & $4.48 \times 10^{-9}$ \\
833 & $2.07 \times 10^{-9}$ & $6.03 \times 10^{-9}$ & $5.08 \times 10^{-9}$ \\
988 & $4.55 \times 10^{-9}$ & $5.92 \times 10^{-8}$ & $4.62 \times 10^{-8}$ \\
1058 & $8.29 \times 10^{-8}$ & $1.02 \times 10^{-6}$ & $8.00 \times 10^{-7}$ \\
1130 & $1.11 \times 10^{-7}$ & $5.00 \times 10^{-7}$ & $4.08 \times 10^{-7}$ \\
1204 & $7.28 \times 10^{-6}$ & $7.83 \times 10^{-6}$ & $7.70 \times 10^{-6}$ \\
1516 & $1.54 \times 10^{-5}$ & $1.52 \times 10^{-5}$ & $1.53 \times 10^{-5}$ \\
\hline
\end{tabular}

\section{Dynamics of Cluster-Molecule Interactions}

We describe here characteristic features of the dynamics of cluster-molecule interactions as derived from quasiclassical trajectory simulations of the $\mathrm{Ni}_{13}+\mathrm{D}_{2}$ 
collision system [55-59]. We restrict our consideration to the ligation process, i.e., to the adsorption of the molecule on the cluster. Simulations show that upon collision with the cluster the molecule may break up, which results in atomic adsorption (direct reaction), or it may get attached to the cluster intact forming a cluster-molecule complex. Each complex, or resonance, has a finite lifetime. A resonance is a reactive one if it leads to an eventual dissociation of the molecule on the cluster (indirect reaction). The nonreactive resonances - the ones that result in desorption rather than dissociation of the molecule - are not considered here. For each fixed set of initial conditions, specified as discussed in Section 3, N=500 to 10000 trajectories are run. These trajectories differ in the initial phase of vibration of $\mathrm{D}_{2}$ and the initial relative orientation of the molecule and of the cluster. The state-specific total reaction probability $\mathrm{P}_{\mathrm{v}, \mathrm{j}, \mathrm{T}, \ldots .}\left(\mathrm{E}_{\mathrm{tr}}, \mathrm{b}\right)$ is calculated in accordance with

$$
P_{v, j, T, \ldots}\left(E_{t r}, b\right)=\frac{\tilde{N}_{v, j, T, \ldots . . .}\left(E_{t r}, b\right)}{N}
$$

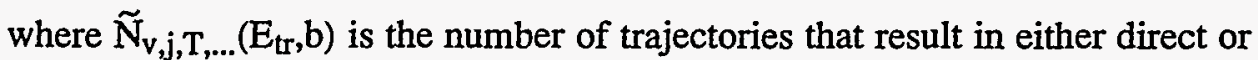
indirect reaction. The probability is computed on grids of $E_{t r}$ and $b$ defined by increments of $\Delta \mathrm{E}_{\mathrm{tr}}=0.05 \mathrm{eV}$ and $\Delta \mathrm{b}=0.25 \AA$, respectively. The state-resolved

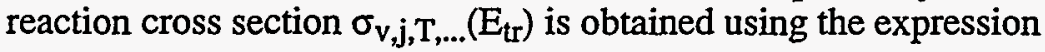

$$
\sigma_{v, j, T, \ldots}\left(E_{t r}\right)=2 \pi \int_{0}^{b_{\max }} b P_{v, j, T}, \ldots\left(E_{t r}, b\right) d b,
$$

where $b_{\max }$ is the maximal impact parameter contributing to the reaction. The

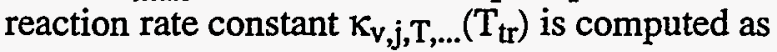

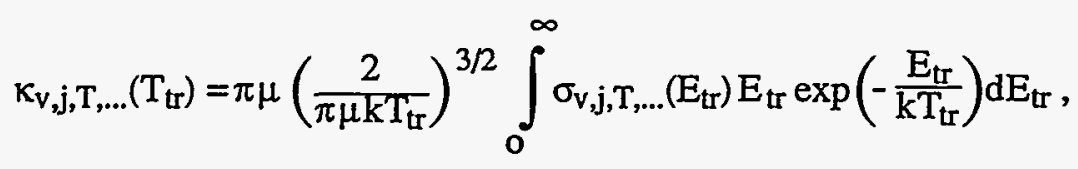

where $T_{t r}$ is the relative translational ("collisional") temperature, and $\mu$ is the reduced mass of the cluster-molecule system.

First, we present and discuss results obtained with the PESII parameterization of the $\mathrm{Ni}_{13}-\mathrm{D}_{2}$ interaction potential. Cross sections of the dissociative adsorption of the molecule on an ico $\mathrm{Ni}_{13}$, initially at room temperature, are shown in Fig. 12. The graphs correspond to different initial rovibrational states of the molecule. The $(v=0, j=10)$ and $(v=1, j=0)$ states are chosen because they are almost degenerate energetically and, therefore, they can be used for testing the mode-selectivity of the reaction. In this case, modeselectivity means sensitivity to the initial partitioning of a given amount of energy between the vibrational and rotational modes of $D_{2}$. The features displayed by the graphs can be summarized as follows. Reactivity of the cluster 


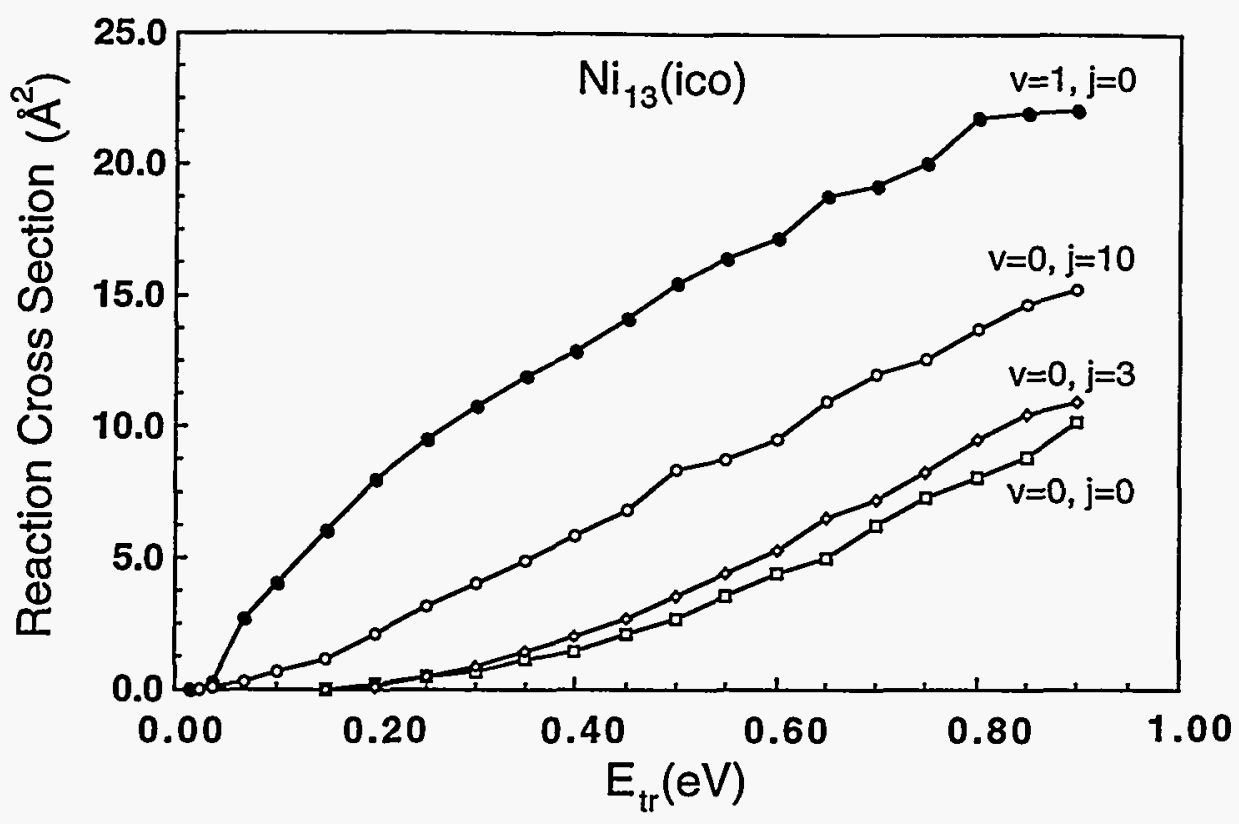

Figure 12. Reaction cross sections as functions of the collision energy for a room-temperature ico $\mathrm{Ni}_{13}$. The different graphs correspond to different initial rovibrational states of $\mathrm{D}_{2}$.

increases monotonically with the collision energy. In order for the reaction to take place the collision energy has to exceed small $(v, j)$-dependent threshold values. Higher initial rovibrational energies of the molecule produce larger reaction cross sections. The reaction is mode-selective: vibrational excitation of the molecule promotes $D-D$ bond breaking more readily than depositing the excess energy into the rotational mode.

Figure 13 displays the dependence of the reaction cross section on the cluster temperature for the ico isomer and $(v=0, j=3)$ initial state of the molecule. The values of the cross section remain practically unchanged in the range $\mathrm{T}=0-298 \mathrm{~K}$. This has been confirmed experimentally [60]. A relatively modest heating causes only small departures of the heavy nickel atoms from their equilibrium icosahedral sites. The surprising feature is that further heating and even melting of the cluster has only a minor effect on its reactivity. One notices that increase of the cluster temperature lowers the $E_{t r}$-threshold to reaction.

Because the cubo and hcp isomers of $\mathrm{Ni}_{13}$ are metastable, they are prepared initially at $T=0 \mathrm{~K}$. The reaction cross sections for these two isomers are shown in Fig. 14. The graphs in the two panels of the figure are strikingly similar, which can be explained by the topological similarity of the cubo and hcp forms of the cluster (cf. Fig. 4). Both possess threefold and fourfold faces, whereas the ico isomer has only threefold faces. The cubo structure is slightly more reactive than the hcp, and both are more reactive than the ico form. As in the case of the ico isomer, higher initial rovibrational energies of the molecule produce larger 


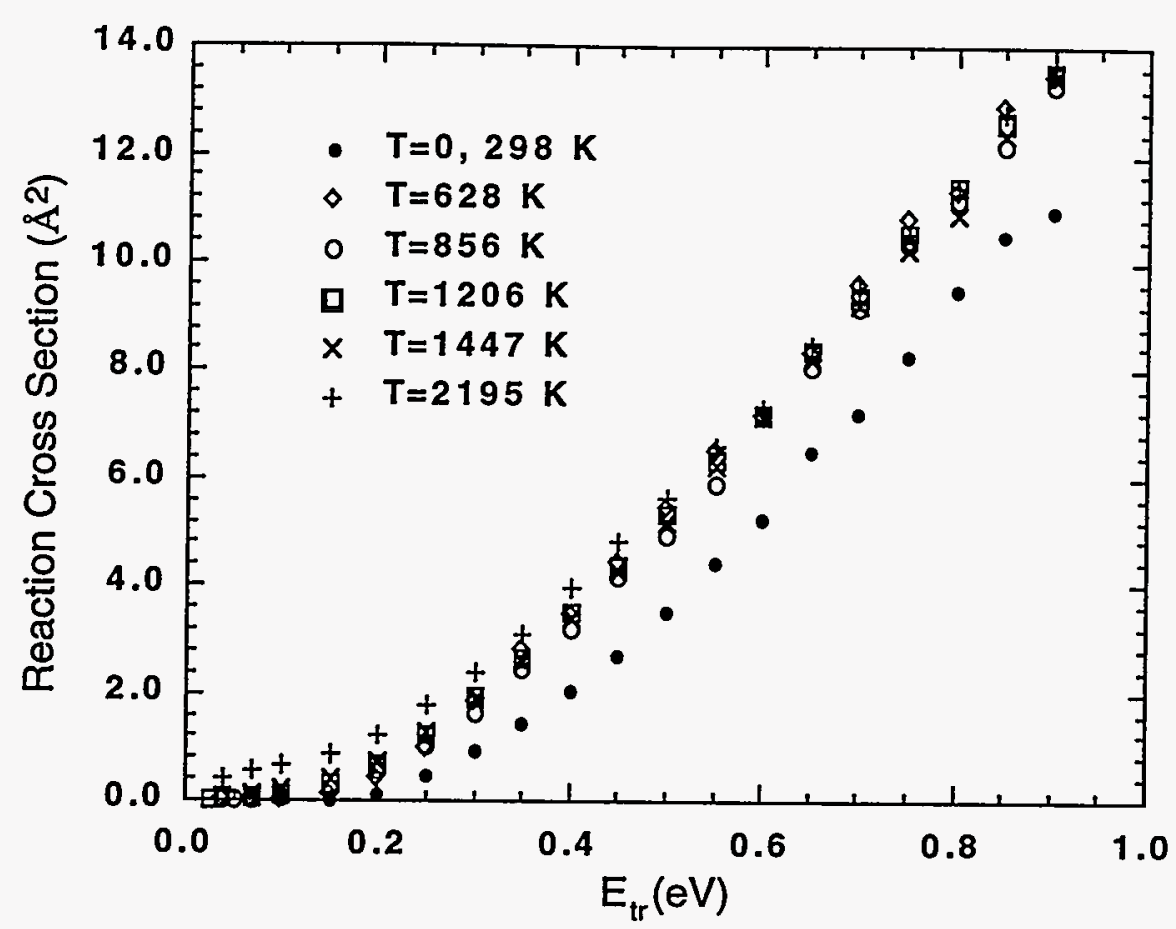

Figure 13. Reaction cross sections as functions of the collision energy for dissociative adsorption of $D_{2}(v=0, j=3)$ on an ico $\mathrm{Ni}_{13}$ at different initial temperatures $\mathrm{T}$ of the cluster.

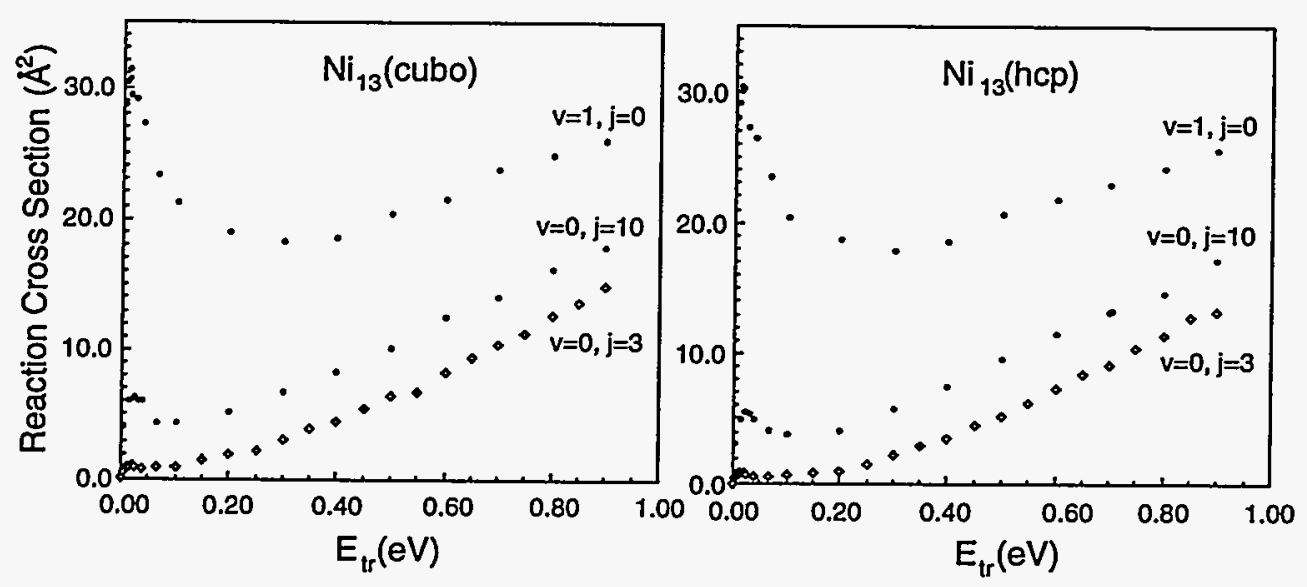

Figure 14. The same as Fig. 12 but for zero-temperature cubo and hep forms of $\mathrm{Ni}_{13}$.

reaction cross sections and the reaction is mode-selective. Graphs of Fig. 14, however, do not display thresholds. Their most distinct feature is a nonmonotonic change of the reaction cross section with the collision energy. At 
high collision energies the reactivity of the cluster becomes a less sensitive function of its structure.

The narrow peaks in the graphs of Fig. 14 at very low collision energies are due almost entirely to reactive resonances. We determined this by applying a scheme that uses time-dependent reaction probabilities and cross sections to distinguish between the direct and indirect reaction pathways and that allows one to compute reactive-resonance formation probabilities (cross sections) and characteristic lifetimes. (The formulation of the scheme and details can be found in $[56,57,59]$.) At very low collision energies the probability (cross section) of molecular adsorption, or resonance formation, is high. This is true for all the structures of $\mathrm{Ni}_{13}$ considered here. A large fraction of those resonances, which involve cubo and hcp isomers, turns out to be reactive. The low- $E_{\text {tr }}$ peaks in the graphs of Fig. 14 are consequences of this fact. The resonances formed at very low collision energies with the ico isomer are nonreactive - the adsorbed molecule eventually desorbs from the cluster without ever dissociating. This is the reason for the absence of low- $E_{t r}$ peaks in the graphs of Fig. 12. An energy-based argument that explains the very different low- $\mathrm{E}_{\mathrm{tr}}$ reactivity of the ico isomer, on the one hand, and the cubo and hcp structures, on the other, is given below. Over a finite range (up to $\sim 0.30 \mathrm{eV}$ ) the reactivity of the cubo and hcp isomers is a decreasing function of $E_{\mathrm{tr}}$. This counterintuitive result is a consequence of the change in the relative contributions of the direct and resonance-mediated reaction pathways with the collision energy. As $E_{t r}$ increases, the probability (cross section) of molecular adsorption decreases and, consequently, so does the contribution of the indirect reaction that is still dominant in this energy range. With further increase of $E_{t r}$ the contribution of the direct pathway begins to offset the losses in the and the reactivity of the cubo and hcp structures of $\mathrm{Ni}_{13}$ becomes an increasing function of the collision energy. The characteristic lifetime of reactive resonances is a decreasing function of $\mathrm{E}_{\mathrm{tr}}$. This is why the fraction of the direct reaction increases with increase of the collision energy. (For details see $[56,57,59]$ ). Recent experiments on reactions of nickel clusters with $D_{2}[60,61]$ corroborate the important role of the resonance-mediated reaction pathway. In at least one respect, however, the experiments disagree with the theoretical predictions. The room-temperature value of the rate of reaction $\mathrm{Ni}_{13}+\mathrm{D}_{2} \rightarrow \mathrm{Ni}_{13} \cdot 2 \mathrm{D}$ computed from PESII is, even for the most reactive isomer of the cluster, orders of magnitude lower than the corresponding value deduced from the measurements [60]. This means that the PESII parameterization of the clustermolecule part of VLEPS has to be modified. We used the following

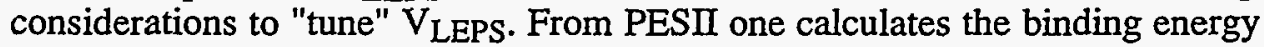
of a D atom in a threefold hollow cite of an ico $\mathrm{Ni}_{13}$ to be $2.43 \mathrm{eV}$, which is $-10 \%$ lower than the measured energy of binding of a $D$ atom to (111) and (100) faces of bulk nickel [61]. The exact value of the $\mathrm{D}-\mathrm{Ni}_{13}$ binding energy is not known at present. However, the possibility that this energy is close to, or even exceeds, the energy of atom-surface binding has been discussed in the literature [62]. Our modification of $V_{\text {LEPS }}$ consists of increasing the PESII well depth of the D-Ni interaction by $10 \%$, which leads to an increase of the $\mathrm{D}-\mathrm{Ni}_{13}$ 
binding energy by $10 \%$. This modification of PESII is certainly within the limits of uncertainty of its original parameters.

Reaction cross sections computed with the modified $V_{\text {LEPS }}$ are shown in Fig. 15. Comparison of the graphs for the ico and cubo isomers with the corresponding graphs of Figs. 12 and 14 shows that the relatively minor change in the cluster-molecule interaction potential causes a dramatic increase in the cluster reactivity. The reaction rate constant calculated for the ico isomer from the cross section of Fig. 15 at $\mathrm{T}_{\mathrm{tr}}=298 \mathrm{~K} \mathrm{is} 3 \cdot 10^{-10} \mathrm{~cm}^{3} / \mathrm{s}$. This value is in good agreement with the estimate of $(8 \pm 4) \cdot 10^{-10} \mathrm{~cm}^{3} / \mathrm{s}$ deduced from experiments [60]. As is clear from Fig. 15, the modified potential eliminates the reaction thresholds for the ico isomer and it allows this isomer to support reactive resonances even at very low collision energies (cf. the peak in the low- $E_{t r}$ range of the corresponding graph). It also virtually eliminates the difference in the reactivity of the ico and cubo forms of the cluster, with the possible exception of very low collision energies where the cubo isomer remains slightly more reactive. Tuning of the potential does not change the earlier conclusion that the (ico) cluster temperature has at most only a weak effect on its reactivity. The only noticeable temperature effect obtained from the modified VLEPS is the gradual disappearance of the characteristic low- $\mathrm{E}_{\mathrm{tr}}$ resonance peak in the graph of the reaction cross section as the temperature of the cluster is increased [63]. This observation reflects the fact that resonance formation probabilities (cross sections) decrease with increase of cluster temperature.

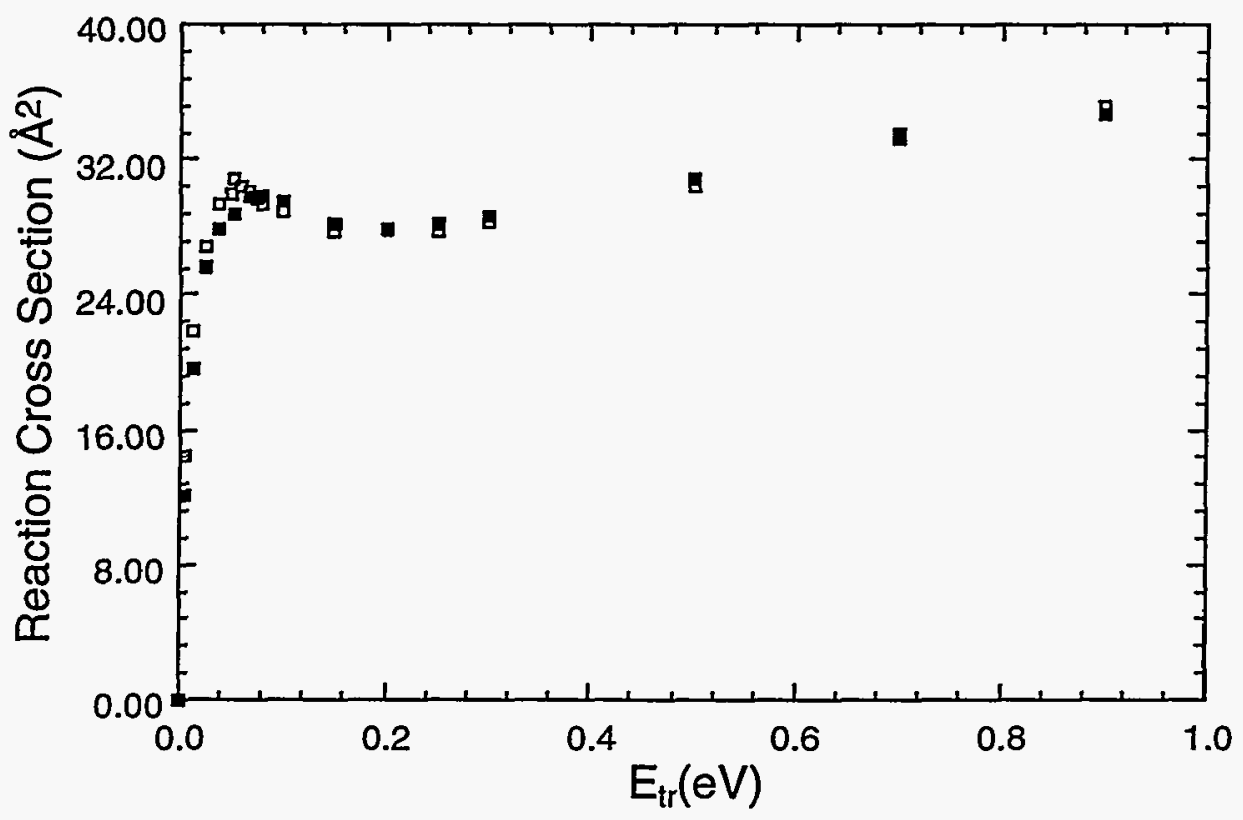

Figure 15. Reaction cross sections for room-temperature ico (full squares) and zerotemperature cubo (empty squares) forms of $\mathrm{Ni}_{13}$ obtained using the modified $\mathrm{V}_{L E P S}$. The graphs correspond to the $(v=0, j=3)$ initial state of $D_{2}$. 
Features of the reactive dynamics described above are ultimately defined by the topology of the cluster-molecule interaction potential, which in this case is 39-dimensional. In order to understand the changes in the dynamics caused by tuning of $V_{\text {LEPS }}$ one must understand how this tuning alters the topology of

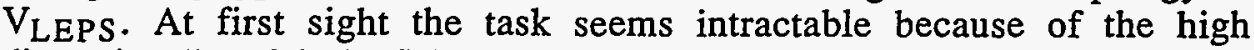
dimensionality of the PES. However, taking into account that adsorption (either molecular or dissociative) of $\mathrm{D}_{2}$ on $\mathrm{Ni}_{13}$ perturbs the equilibrium structure of the cluster only negligibly (e.g., the energy of the equilibrium ico configuration changes by less than $0.01 \%$ ), one realizes that the cluster degrees of freedom can be treated as frozen. This reduces the dimensionality of the problem to six. The six degrees of freedom are those of the two D atoms. In the context of our discussion, the two important degrees of freedom are the distance between the $\mathrm{D}$ atoms and the distance between the COMs of $\mathrm{Ni}_{13}$ and $\mathrm{D}_{2}$. One can map out the cluster-molecule interaction potential as a function of these two distances by minimizing it over the remaining four degrees of freedom, which we choose as four angles that specify the orientation of the two distances in space.

The upper panel of Fig.16 shows the original (PESII) $V_{\text {LEPS }}$ mapped out in this way for the ico isomer of the cluster and presented in the form of equipotential contour plots (ECP). At large separations between $\mathrm{D}_{2}$ and $\mathrm{Ni}_{13}$ the depth of the entrance valley is $4.74 \mathrm{eV}$, which is the depth of the D-D Morse potential. As the molecule approaches the cluster, a shallow well develops that corresponds to the state of molecular adsorption. In fact, the bottom of the well corresponds to two energetically degenerate configurations: the molecule is parallel to a face of the cluster and is either parallel or perpendicular to an edge of that face. The minimum energy path then leads in the direction of increasing D-D distances. The barrier (saddle) for the dissociation of the molecule is a late one. This is why vibrational excitation of $\mathrm{D}_{2}$ is so efficient in promoting the reaction. However, moving toward and across the dissociation barrier also involves simultaneous further movement of the molecule toward the cluster. This explains the role of the collision energy in the reaction. One notices that the contour of energy $-4.55 \mathrm{eV}$ (this is the energy of $\mathrm{D}_{2}$ with its zero-point vibration included) does not reach the dissociation barrier along either of the two distance coordinates. This is why the collision energy must exceed a minimum (threshold) value to initiate the reaction and why the resonances formed at very low collision energies are nonreactive. The dissociation barrier is followed by five wells of approximately equal depth separated by barriers (saddles) of approximately equal height. These wells represent the five possible topologically different pairs of threefold faces of an ico 13-mer as the energetically favorable sites for adsorption of the two $D$ atoms. The well corresponding to the largest D-D separation represents adsorption on the opposite faces of the cluster. The barriers between the wells are the atomic diffusion barriers. They correspond to one of the two $\mathrm{D}$ atoms being over a face and the other over an edge of the cluster. The specific features of the reactivity of the cubo and hcp forms of $\mathrm{Ni}_{13}$ can be analyzed in a similar way. Here we mention only that the energy of $\mathrm{D}_{2}$ with its zero-point vibration included is sufficient to surmount the dissociation barrier for these forms of the cluster. 

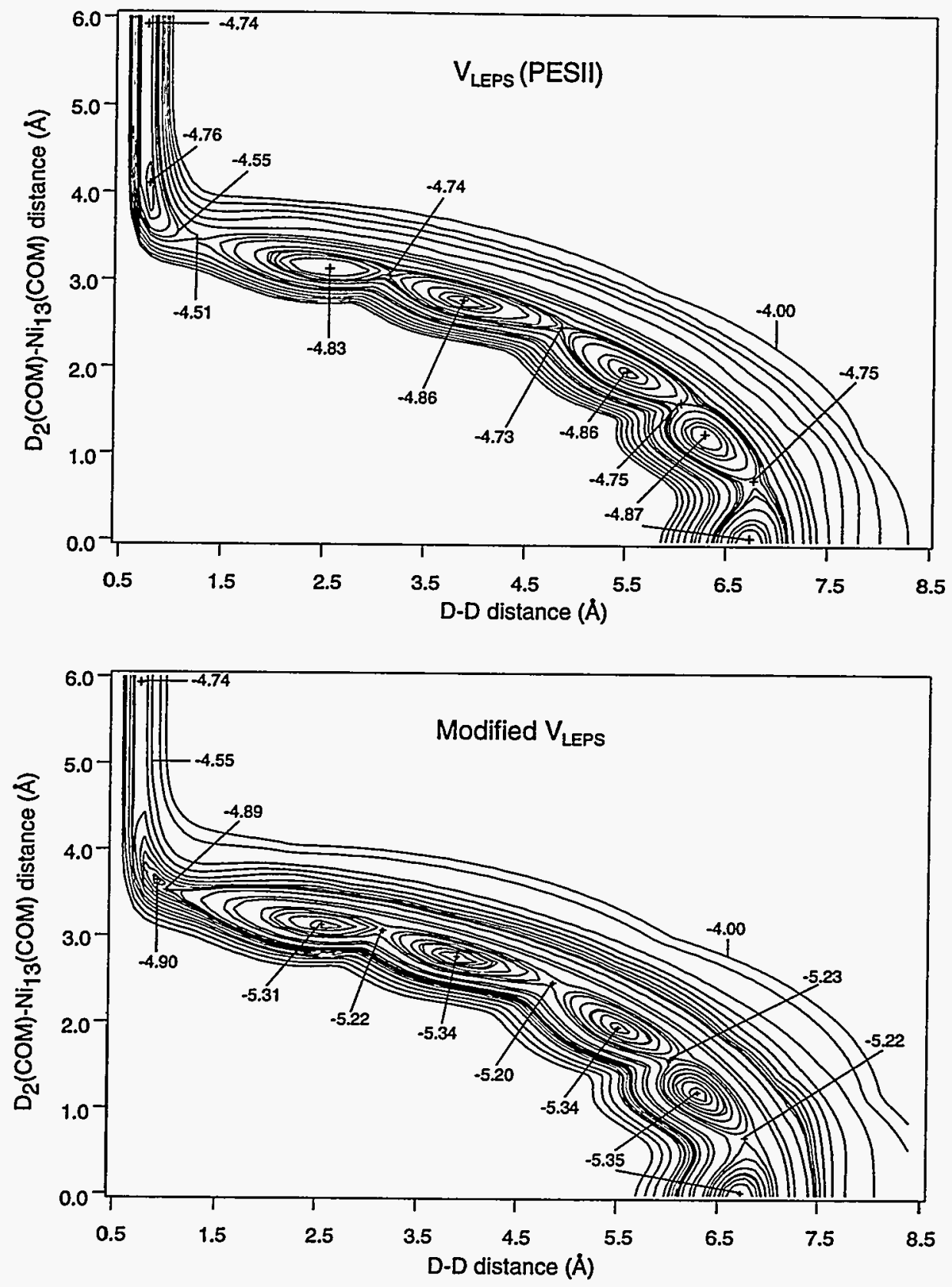

Figure 16. Equipotential contour plots for the original (PESI) and modified $\mathrm{V}_{\text {LEPS. }}$ The crosses indicate minima and maxima on the surfaces. The numbers are values of energy in $\mathrm{eV}$. 
The ECP map of the modified VLEPS is shown for the ico $\mathrm{Ni}_{13}$ in the lower panel of Fig. 16. Comparison of the original and modified cluster-molecule interaction potentials shows that their exit valley parts are essentially identical, except of a uniform $10 \%$ disparity in the magnitude of the corresponding energies. The major difference between the two is in the transition region connecting the entrance and the exit valleys. The molecular adsorption well in the modified $V_{\text {LEPS }}$ moves to slightly larger D-D separations, and it is supported from the exit valley side only by a very small local saddle. The energy of this saddle is lower than that of the bottom of the entrance valley, which means that the molecule does not experience any configurational energy barrier to dissociation. It may dissociate on the cluster irrespective of how slowly it approaches it. This explains why the modified $V_{\text {LEPS }}$ produces a considerably higher reactivity of the ico isomer of the cluster.

In order to further compare the original and the modified potentials we calculated the vibrational frequencies of the $\mathrm{D}$-cluster and $\mathrm{D}_{2}$-cluster bonds when the atom and the molecule are adsorbed on a face of an ico $\mathrm{Ni}_{13}$. For a $\mathrm{D}$ atom the original potential gives $754 \mathrm{~cm}^{-1}$, the modified $-791 \mathrm{~cm}^{-1}$. For an $\mathrm{H}$ atom these frequencies translate into $1066 \mathrm{~cm}^{-1}$ and $1123 \mathrm{~cm}^{-1}$, respectively. Both values are close to the measured $\left(1122-1137 \mathrm{~cm}^{-1}\right.$ [64]) and calculated (1043-1176 cm $\left.\mathrm{cm}^{-1}[62,65]\right)$ frequencies of vibration of an $\mathrm{H}$ atom in a threefold hollow site of the (111) face of bulk nickel. For the molecular adsorption the original $V_{\text {LEPS }}$ gives $138 \mathrm{~cm}^{-1}$, whereas the modified $-382 \mathrm{~cm}^{-1}$. The two values are sufficiently different to allow, at least in principle, for an experimental testing of the two potentials.

\section{Summary}

We gave a brief outline of the $\mathrm{MD}$ technique as an efficient tool for theoretical studies of atomic clusters and presented a sample of results on metal clusters derived from trajectory simulations. The issues discussed include structural properties, intracluster dynamics over a broad range of internal energy, and interactions of clusters with molecules.

The power of the MD technique is that it allows one to simulate real-time evolution of physical systems - clusters in our case - and thereby to understand and characterize the fundamental mechanisms that define and govern their properties. An important component of any MD study is the description of the interatomic interactions. The degree of adequacy of this description defines the ultimate reliability of $\mathrm{MD}$ results. Ideally, one would like to use high level first principles calculations to arrive at energies of interatomic interactions and forces acting on atoms. The continuing progress in computer technology and computational methodologies make this goal ever more realistic. However, for many-electron systems, such as, e.g., transition metals, the task remains prohibitively costly even for small size clusters. And even when a first principles treatment is feasible for one or a few fixed nuclear configurations, it may not be efficient enough to allow for large scale dynamical simulations. 
An alternative is to use fitted semiempirical potentials. Improving the quality of these potentials, both for bare metal clusters and cluster-molecule systems, is a task of paramount importance. It is expected that an increasing amount of relevant first principles and experimental data will become available for performing the fits. For example, it would be very useful to have experimental information on the structure-reactivity correlation (or lack of it) in reaction of $\mathrm{D}_{2}$ with $\mathrm{Ni}_{13}$ and on the vibrational frequency of the cluster-molecule bond. This information could be used for further calibration of the cluster-molecule interaction potential discussed in the previous section. An intimate interplay between theory and experiment is important not only as a tool for testing and validating the results but also as a means of achieving a better understanding of the fundamental interatomic interactions that are ultimately responsible for all the observed properties.

\section{Acknowledgments}

The work presented was performed under the auspices of the Office of Basic Energy Sciences, Division of Chemical Science, US-DOE under contract number W-31-109-ENG-38. Different parts of the work were carried out in collaboration with my colleagues and former postdocs Drs. I. L. Garzón, Z. B. Güvenç, D. H. Li, and M. J. López, to whom I express my appreciation.

\section{References}

1. P. Jena, B.K. Rao, and S.N. Khanna (eds) (1987) Physics and Chemistry of Small Clusters, Plenum Press, New York.

2. I. Prigogine and S.A. Rice (eds) (1988) Adv. Chem. Phys. Vol. 10, Part 2, WileyInterscience, New York.

3. G. Benedek, T.P. Martin, and G. Pacchioni (eds) (1988) Elemental and Molecular Clusters, Springer-Verlag, Berlin.

4. P. Jena, S.N. Khanna, and B.K. Rao (eds) (1992) Physics and Chemistry of Finite Systems: From Clusters to Crystals, Vols. 1 and 2, Kluwer Academic Publishers, Dordrecht.

5. R. Schmidt, H.O. Lutz, and R. Dreizler (eds) (1992) Nuclear Physics Concepts in the Study of Atomic Cluster Physics, Lecture Notes in Physics, Vol. 404, Springer-Verlag, Berlin.

6 H. Haberland (ed) (1994) Clusters of Atoms and Molecules, Springer Series in Chemical Physics, Vol. 52, Springer-Verlag, Berlin.

7. H. Haberland (ed) (1994) Clusters of Atoms and Molecules II, Springer Series in Chemical Physics, Vol. 56, Springer-Verlag, Berlin.

8. R. Car and M. Parrinello (1985) Phys. Rev. Lett. 55, 2471.

9. R.N. Barnett, U. Landman, A. Nitzan and G. Rajagopal (1991) J. Chem. Phys. 94, 608; J.C. Greer, R. Ahlrichs, and I.V. Hertel (1991) Z. Phys. D 18, 413; S.A. Maluendes and M. Dupuis (1992) Int. J. Quant. Chem. 42, 1327; G. Kresse and J. Hafner (1993) J. Non-Cryst. Solids 156-158, 956; B. Hartke, D.A. Gibson, and E.A. Carter (1993) Int. J. Quant. Chem. 45, 59; B. Hartke and E.A. Carter (1993) Chem. Phys. Lett. 216, 324; and references therein.

10. U. Röthlisberger and W. Andreoni (1991) J. Chem. Phys. 94, 8129. 
11. J. Jellinek, V. Bonačic-Koutecký, P. Fantucci, and M. Wiechert (1994) J. Chem. Phys. 101, 10092.

12. H. Goldstein (1950) Classical Mechanics, Addison Wesley Publishing Company, Reading.

13. W.H. Press, S.A. Teukolsky, W.T. Vetterling, and B.P. Flannery (1992) Numerical Recipes, Cambridge University Press, Cambridge.

14. J. Jellinek and D.H. Li (1989) Phys. Rev. Lett. 62, 241.

15. D.H. Li and J. Jellinek (1989) Z. Phys. D 12, 177.

16. G. Nyman, S. Nordholm, and H.W. Schranz (1990) J. Chem. Phys. 93, 6768.

17. J.D. Louck and H.W. Galbraith (1976) Rev. Mod. Phys. 48, 69.

18. J. Jellinek and D.H. Li (1990) Chem. Phys. Lett. 169, 380.

19. A. Münster (1969) Statistical Thermodynamics, Academic Press, New York.

20. J. Jellinek (1988) J. Phys. Chem. 92, 3163.

21. J. Jellinek and R.S. Berry (1989) Phys. Rev. A 40, 2816.

22. A.I. Khinchin (1969) Mathematical Foundations of Statistical Mechanics, Dover, New York.

23. S. Nosé (1984) J. Chem. Phys. 81, 511; S. Nosé (1984) Mol. Phys. 52, 244.

24. W.G. Hoover (1985) Phys. Rev. A 31, 1695; H.A. Posch, W.G. Hoover, and F.J. Vesely (1985) Phys. Rev. A 33, 4253.

25. S. Nosé (1991) Prog. Theor. Phys. Suppl. $103,1$.

26. M.S. Daw and M.I. Baskes (1984) Phys. Rev. B 29, 6443.

27. A.F. Voter and S.P. Chen, (1987) Mater. Res. Soc. Symp. 82, 175.

28. R.P. Gupta (1981) Phys. Rev. B 23, 6265.

29. F. Ducastelle (1970) J. Phys. (Paris) 31, 1055.

30. V. Rosato, M. Guillope, B. Legrand (1989) Philos. Mag. A 59, 321.

31. J. Jellinek and I.L. Garzón (1991) Z. Phys. D 20, 239. Values of kinetic energies and of temperature in this paper and in Refs. [32-34] below have to be divided by two. This does not affect the validity of the qualitative conclusions.

32. I.L. Garzón and J. Jellinek (1991) Z. Phys. D 20, 235.

33. I.L. Garzón and J. Jellinek (1992), in P. Jena, S.N. Khanna, and B.K. Rao (eds), Physics and Chemistry of Finite Systems: From Clusters to Crystals, Vol. 1, Kluwer Academic Publishers, Dordrecht, p. 405.

34. I.L. Garzón and J. Jellinek (1993) Z. Phys. D 26, 316.

35. B. Carnahan, H.A. Luther, and J.O. Wilkes (1969) Applied Numerical Methods, John Wiley \& Sons, New York.

36. L. Verlet (1967) Phys. Rev. 159, 98.

37. T.N. Truong, D.G. Truhlar, and B.C. Garrett (1989) J. Phys. Chem. 93, 8227.

38. K. Raghavan, M.S. Stave, and A.E. DePristo (1989) J. Chem. Phys. $91,1904$.

39. R.N. Porter, L.M. Raff, and W.H. Miller (1975) J. Chem. Phys. 63, 2214.

40. Z.B. Güvenç, J. Jellinek, and A.F. Voter (1992), in P. Jena, S.N. Khanna, and B.K. Rao (eds), Physics and Chemistry of Finite Systems: From Clusters to Crystals, Vol. 1, Kluwer Academic Publishers, Dordrecht, p. 411.

41. M.J. López, and J. Jellinek (1994) Phys. Rev. A 50, 1445.

42. M.S. Stave and A.E. DePristo (1992) J. Chem. Phys. 97, 3386.

43. E.K. Parks, L. Zhu, J. Ho, and S.J. Riley (1994) J. Chem. Phys. 100, 7206.

44. J. Farges, M.F. de Feraudy, B. Raoult, and G. Torchet (1987), in J. Jortner, A. Pullman, and B. Pullman (eds), Large Finite Systems, D. Reidel Publishing Company, Norwell, p. 113.

45. G. Torchet, J. Farges, de M.F. Feraudy, and B. Raoult (1989) Ann. Phys. Fr. 14, 245.

46. C.L. Cleveland and U. Landman (1991) J. Chem. Phys. 94, 7376.

47. B. Raoult, J. Farges, M.F. de Feraudy and G. Torchet (1989) Z. Phys. D 12, 85 .

48. Z.B. Güvenç and J. Jellinek (1993) Z. Phys. D 26, 304.

49. J. Jellinek, T.L. Beck, and R.S. Berry (1986) J. Chem. Phys. 84, 2783.

50. H.L. Davis, J. Jellinek, and R.S. Berry (1986) J. Chem. Phys. 84, 6456. 
51. R.S. Berry, J. Jellinek, and G. Natanson (1984) Phys. Rev. A 30, 919.

52. I.Z. Fisher (1966) Statistical Theory of Liquids, University of Chicago Press, Chicago.

53. H.P. Cheng and R.S. Berry (1992) Phys. Rev. A 45, 7969.

54. F. Ercolessi, W. Andreoni, and E. Tosatti (1991) Phys. Rev. Lett. 66, 911.

55. J. Jellinek and Z.B. Güvenç (1991), in J. Jortner, R.D. Levine, and B. Pullmann (eds), Mode Selective Chemistry, Kluwer Academic Publishers, Dordrecht, p. 153.

56. J. Jellinek and Z.B. Güvenç (1992), in P. Jena, S.N. Khanna, and B.K. Rao (eds), Physics and Chemistry of Finite Systems: From Clusters to Crystals, Vol. 2, Kluwer Academic Publishers, Dordrecht, p. 1047.

57. J. Jellinek and Z.B. Güvenç (1992), in R. Schmidt, H.O. Lutz, and R. Dreizler (eds), Nuclear Physics Concepts in the Study of Atomic Cluster Physics, Lecture Notes in Physics, Vol. 404, Springer-Verlag, p. 169.

58. J. Jellinek and Z.B. Güvenç (1993) $Z$. Phys. D 26, 110.

59. J. Jellinek and Z.B. Güvenç (1994), in B. Remaud, A. Calboreanu and V. Zoran (eds), Topics in Atomic and Nuclear Collisions, Plenum Press, New York, p. 243.

60. L. Zhu, J. Ho, E.K. Parks, and S.J. Riley (1993) J. Chem. Phys. 98, 2798.

61. L. Zhu, J. Ho, E.K. Parks, and S.J. Riley (1993) Z. Phys. D 26, 313.

62. P. Mlynarski and D.R. Salahub (1991) J. Chem. Phys. 95, 6050.

63. Z.B. Güvenç and J. Jellinek, to be published.

64. W. Ho, N.J. DiNardo, and D.E. Plummer (1980) J. Vac. Sci. Technol. 17, 314; R.R. Cavanagh, R.D. Kelley, and J.J. Rush (1982) J. Chem. Phys. 77, 1540.

65. H. Yang and J.L. Whitten (1993) J. Chem. Phys. 98, 4039; and references therein. 